\title{
A mesh-dependent model for applying dynamic contact angles to VOF simulations
}

\author{
S. Afkhami ${ }^{\text {a,* }}$, S. Zaleski ${ }^{\text {b }}$, M. Bussmann ${ }^{c}$ \\ ${ }^{a}$ Department of Mathematics and ICAM, 460 McBryde Hall, Virginia Tech, Blacksburg, VA 24061-0123, USA \\ ${ }^{\mathrm{b}}$ Institut Jean Le Rond D'Alembert, Case 162, Université Pierre et Marie Curie, 75252 Paris Cédex 05, France \\ ${ }^{\mathrm{c}}$ Department of Mechanical and Industrial Engineering, University of Toronto, 5 King's College Road, Toronto, Canada M5S 3G8
}

\section{A R T I C L E I N F O}

\section{Article history:}

Received 8 April 2008

Received in revised form 10 March 2009

Accepted 15 April 2009

Available online 3 May 2009

\section{Keywords:}

VOF

Contact line

Contact angle

Dynamic contact angle

Slip length

\begin{abstract}
A B S T R A C T
Typical VOF algorithms rely on an implicit slip that scales with mesh refinement, to allow contact lines to move along no-slip boundaries. As a result, solutions of contact line phenomena vary continuously with mesh spacing; this paper presents examples of that variation. A mesh-dependent dynamic contact angle model is then presented, that is based on fundamental hydrodynamics and serves as a more appropriate boundary condition at a moving contact line. This new boundary condition eliminates the stress singularity at the contact line; the resulting problem is thus well-posed and yields solutions that converge with mesh refinement. Numerical results are presented of a solid plate withdrawing from a fluid pool, and of spontaneous droplet spread at small capillary and Reynolds numbers.
\end{abstract}

Published by Elsevier Inc.

\section{Introduction}

Dynamic contact line phenomena involve two immiscible viscous fluids in contact with a solid surface; as one of the fluids displaces the other, the contact line moves along the solid surface. Applications range from droplet spreading in various applications to coating flows. Such flows have been studied both theoretically and experimentally (e.g. [1-6]). Simulating such flows is complicated by the mathematical paradox of a contact line moving along a no-slip solid surface. Analytical solutions of the Navier-Stokes equations lead to a stress singularity at the contact line [1,3]. This non-physical divergent stress stems from the fact that continuum fluid mechanics breaks down at molecular distances from the contact line; to circumvent the stress singularity, the molecular interactions between the fluids and the solid would have to be modeled.

Various models have been proposed to deal with the contact line singularity in the context of continuum mechanics. Precursor models assume that a pre-existing thin liquid film covers the surface ahead of the contact line, so that the no-slip condition can be applied over the entire solid surface [7-9]; these models are particularly suited for highly wetting situations. Diffuse interface models treat the interface as a finite layer across which fluid properties vary abruptly but smoothly [10-12]; the contact line slips due to the diffusive fluxes between the fluids. Finally, slip models are used to relax the stress singularity at the contact line [1,2,13,14]; these allow independent imposition of a non-zero contact angle and are suitable for partially wetting contact lines that are advancing or receding over a solid surface.

Many studies have made use of a slip model. For example, Huh et al. [15] applied a free-slip model near the contact line and a no-slip condition elsewhere. Dussan [16] concluded that when compared with the slip length, the details of a partic-

\footnotetext{
* Corresponding author. Tel.: +1 540231 6531; fax: +1 5402315960 .

E-mail address: afkhamis@math.vt.edu (S. Afkhami).
} 
ular slip mechanism are of little importance. Cox [13] also considered slip in his model: the results of his model agreed well with the experimental data of Hoffman [17]. The most common slip model in the literature is the Navier-slip law, in which the extent of slip is proportional to the shear stress

$$
\left.v\right|_{x=0}-U=\left.\lambda \frac{\partial v}{\partial x}\right|_{x=0}
$$

at the solid boundary $(x=0) ; \lambda$ is the "slip length" that defines the extent to which the no-slip boundary is relaxed; $v$ is the slip velocity; and $U$ is the speed at which the solid boundary is moving.

Numerically, the approach to implementing slip depends on the method used to represent the interface. Methods that explicitly track an interface (e.g. [18-20]) require a slip condition for the mesh node at the contact line. On the other hand, most VOF implementations (and more generally, most interface capturing techniques) utilize cell face normal velocities to advect volume fractions (e.g. [21-23]). This implies that the methodology includes an "implicit" (or "effective") slip length at no-slip boundaries. However, relying on this implicit slip length leads to a convergence breakdown, because the stress singularity at the contact line is resolved by a slip length that is proportional to mesh spacing $[22,24,25]$.

An important property of contact line phenomena is the contact angle, the angle at which an interface appears to intersect with a solid boundary. The static contact angle $\theta_{e}$ is the angle that is obtained on a perfectly flat surface in thermodynamic equilibrium, given by the Young relation [7]

$$
\gamma_{s l}+\gamma \cos \theta_{e}=\gamma_{s g}
$$

For example, for a liquid/gas/solid system, $\gamma$ is the liquid/gas surface tension, and $\gamma_{s l}$ and $\gamma_{s g}$ are the surface energies per unit area of the wet and dry solid, respectively. It is often assumed that even in dynamic situations the contact angle at the smallest scale is the angle given by the Young relation (Eq. (2)), although in some cases the local value of the surface tension may differ from its equilibrium value so that the local angle will not be $\theta_{e}$. For instance, $\gamma$ will differ from its equilibrium value if the fluid motion affects the distribution of tensio-active molecules on the interface; other theories involving variable surface tensions have also been proposed [26]. Heterogeneities or roughness of the interface affect the observed contact angles at scales larger than that of the surface imperfections. A dynamic contact angle at this scale will then not be given by Eq. (2), but will result from a complex balance of small scale dissipation and energy release.

Fig. 1 illustrates a moving contact line and three contact angles measured at different distances from the contact line: the microscopic contact angle, $\theta_{\text {micro }}$, the dynamic contact angle, $\theta_{\text {dyn }}$, and the apparent contact angle, $\theta_{\text {app }} \cdot \theta_{\text {micro }}$ is measured at the microscopic length scale and here is equivalent to $\theta_{e} \cdot \theta_{\text {dyn }}$ is the contact angle determined by measuring the interface slope at the contact line; this is the angle often used as a geometric boundary condition for the solution of hydrodynamic equations. $\theta_{a p p}$, the interface slope at a finite distance from the contact line, is used as an auxiliary concept to interpret experimental or numerical results. For example, for a meniscus advancing in a capillary tube or for a plate withdrawing from a liquid pool, $\theta_{a p p}$ is the angle between a spherical cap fitted to the moving interface and the solid substrate; for a spreading droplet, $\theta_{a p p}$ can be determined by fitting a static-like interface to the drop profile near the moving contact line.

When a contact line moves across a solid surface, there are three forces (assuming gravity is negligible) acting on the fluid: inertial, viscous, and surface tension. Theoretical studies suggest that the viscous dissipation becomes very strong near a moving contact line [1]. The resulting interaction between hydrodynamic and surface tension forces then yields a curvature of the fluid/fluid interface that varies sharply near the contact line. This can cause the contact angle to differ significantly from the interface angle measured even at short distances away from the contact line. The magnitude of this effect depends on fluid properties, the capillary number $\mathbf{C a}=\mu U_{c l} / \gamma\left(U_{c l}\right.$ is the contact line velocity, $\mu$ the fluid viscosity, and $\gamma$ the surface

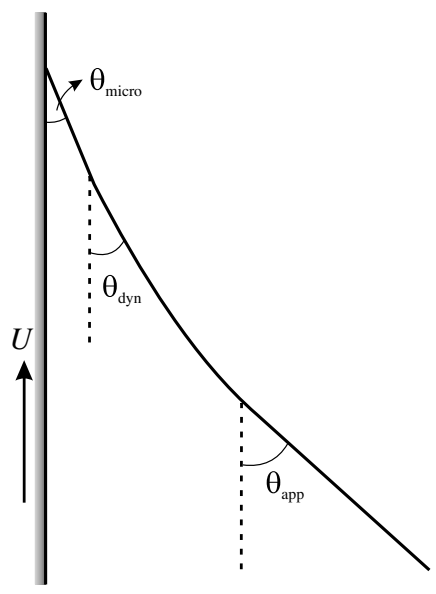

Fig. 1. A schematic of different angles at a moving contact line. 
tension), and a contact line region length scale, possibly of molecular size [27]. A successful example of determining apparent contact angles from hydrodynamic theories is the computational study of Lowndes [28] who used a finite-element method to solve for single-phase capillary tube flow with local slip and a very small slip length. Lowndes demonstrated a dependence of $\theta_{a p p}$ on Ca and on the static contact angle that agreed well with several experimental studies. Sheng et al. [29] predicted the interface profiles in a capillary tube, solving the time-independent Stokes equations with three different slip models. They too showed a dependence of $\theta_{\text {app }}$ on Ca, the static contact angle, and $\lambda$ that agreed well with theoretical expressions. Numerical results of Latva-Kokko and Rothman [30] obtained from a lattice-Boltzmann model also showed the dependence of $\theta_{\text {app }}$ on Ca and $\lambda$ that is predicted by analytical relations.

Theoretical studies also suggest that at low Ca, there exists a viscous region close to the contact line where the flow is geometry-free. Numerical models have been proposed with the aim to provide geometry-independent boundary conditions for modeling of dynamic contact lines (for review, see [31]). Specifically, it has been suggested that the viscous region around the contact line can be cut off from the flow (to avoid the singularity at the contact line) and be replaced with the flow given by the analytical solution. Bazhlekov and Chesters [32] and Somalinga and Bose [33] utilized this strategy and showed that for $\mathbf{C a}<0.1$, the results compared well with the results of numerical simulations which resolved the microscopic scale.

This work presents VOF-based numerical solutions of the time-dependent Navier-Stokes equations for flows with dynamic contact lines. We solve for the stationary state interface profiles of a receding contact line (withdrawing plate), and transient interface profiles of an advancing contact line (spreading droplet). We first demonstrate the dependence of the results on mesh refinement, when a no-slip or Navier-slip condition is imposed along the solid boundary, and a fixed contact angle is imposed at the contact line. Then we present a brief review of the theoretical analysis of Cox [13], that forms the basis for the development of our contact angle model. Finally we present simulation results using the new contact angle model, that converge with mesh refinement.

\section{Numerical methodology}

We solve the equations of conservation of mass and momentum for two incompressible Newtonian fluids. Each fluid has a constant density $\rho$ and a constant viscosity $\mu$. In what follows, $\mathbf{u}$ represents the velocity field, $p$ the hydrodynamic pressure, and $g$ the gravitational acceleration. We non-dimensionalize the variables as follows:

$$
\begin{aligned}
& \mathbf{x}^{*}=\mathbf{x} / L, \quad t^{*}=t U_{0} / L, \quad \mu^{*}=\mu / \mu_{0}, \quad \rho^{*}=\rho / \rho_{0}, \\
& \mathbf{u}^{*}=\mathbf{u} / U_{0}, \quad p^{*}=p L /\left(\mu_{0} U_{0}\right)
\end{aligned}
$$

where $L, U_{0}, \rho_{0}$, and $\mu_{0}$ are characteristic length, velocity, density, and viscosity, respectively. The equations of motion then become

$$
\begin{aligned}
& \nabla^{*} \cdot \mathbf{u}^{*}=0 \\
& \operatorname{Re}\left(\frac{\partial}{\partial t^{*}}\left(\rho^{*} \mathbf{u}^{*}\right)+\nabla^{*} \cdot\left(\rho^{*} \mathbf{u}^{*} \mathbf{u}^{*}\right)\right)=-\nabla^{*} p^{*}+\nabla^{*} \cdot \tau^{*}+\frac{1}{\mathbf{C a}} \mathbf{F}_{s t}^{*}+\rho^{*} \frac{\mathbf{B o}}{\mathbf{C a}}
\end{aligned}
$$

where $\tau^{*}=\mu^{*}\left(\nabla^{*} \mathbf{u}^{*}+\nabla^{*} \mathbf{u}^{* T}\right)$ represents the dimensionless shear stress tensor, $\mathbf{F}_{s t}^{*}$ denotes the dimensionless body force due to surface tension, and the Reynolds, capillary, and Bond numbers are defined as $\mathbf{R e}=\rho_{0} U_{0} L / \gamma, \mathbf{C a}=\mu_{0} U_{0} / \gamma$, and Bo $=\rho_{0} L^{2} g / \gamma$, respectively.

The "volume-of-fluid" (VOF) method is used to track the interface [34]. A balanced-force implementation of the "continuum surface force" (CSF) method [35,36] is used to discretize the surface tension force. The "height function" (HF) methodology [36,37] is implemented for calculating interface normals and curvatures and for imposing a contact angle as a boundary condition at the contact line [37].

The basis for our in-house numerical model is an early version of GERRIS [38,39]. The flow equations are discretized using a projection method based on a variable-density fractional-step scheme. (However, all the results in this paper are all of constant density interfacial flows.) The domain is discretized adaptively using quadtree finite volumes arranged hierarchically. Primitive variables are collocated at cell centers; normal velocities are also specified at faces (MAC scheme). Advection terms are discretized using a second-order upwind scheme. In the fractional-step projection method, an interim velocity is computed first; this velocity field is projected onto a divergence-free velocity field, with the pressure field obtained as the solution of a Poisson equation. As the face-centered velocities are exactly divergence-free, the volume fractions are advected using these velocities.

As mentioned previously, for most VOF implementations including ours, explicit velocity boundary conditions are not required at a contact line. Instead, volume fractions in cells that contain a contact line are advected with the (non-zero) velocities that are a half cell from the solid boundary, as illustrated in Fig. 2.

\section{Mesh-dependent results, applying no-slip and Navier-slip boundary conditions}

We begin by presenting results that depend on mesh resolution, when the no-slip or the Navier-slip boundary condition is applied at the solid surface along which a fluid/fluid interface moves. Fig. 3 illustrates the implementation of the boundary 


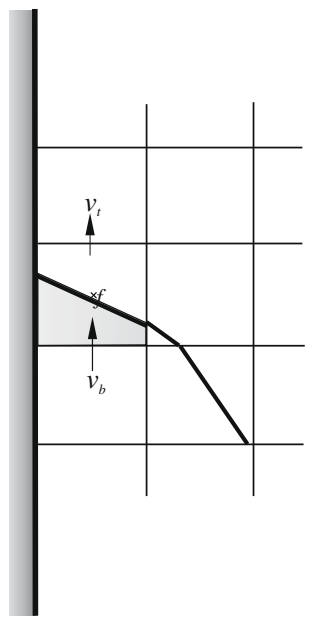

Fig. 2. VOF methods use cell face normal velocities, $v_{b}$ and $v_{t}$, to advect the cell center volume fraction $f$, and so move the contact line.

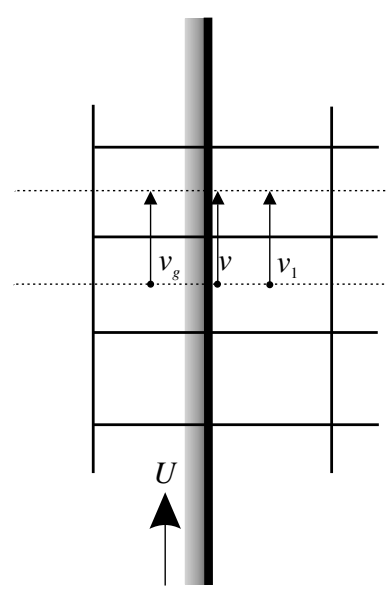

(a)

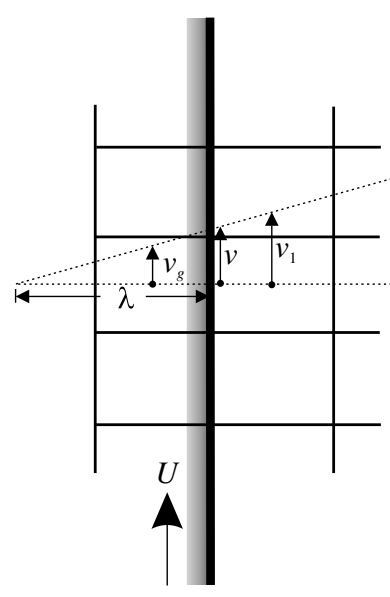

(b)

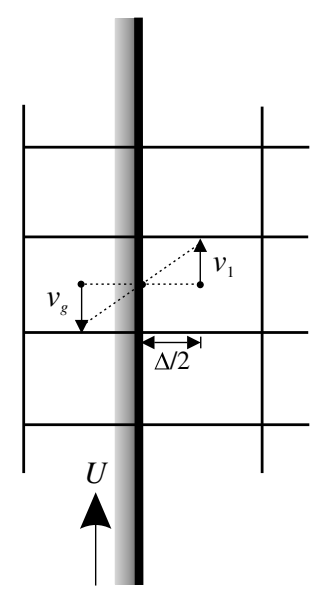

(c)

Fig. 3. The discretization of Eq. (1): (a) $\lambda \rightarrow \infty$, (b) $0<\lambda<\infty$, and (c) $\lambda=0(v=0$ ).

conditions, by specifying velocities in the so-called "ghost cells" just outside the fluid domain. The Navier-slip model (Eq. (1)) is discretized using central differences so that the ghost cell velocity is

$$
v_{g}=\frac{2 U \Delta-(\Delta-2 \lambda) v_{1}}{\Delta+2 \lambda}
$$

and the slip velocity $v$ along the solid boundary is

$$
v=\frac{U \Delta+2 \lambda v_{1}}{\Delta+2 \lambda}
$$

where $v_{1}$ is the component of the tangential fluid velocity one half cell width from the wall (illustrated in Fig. 3), and $\Delta$ is the (uniform) mesh size. Note that $\lambda=\infty$ (Fig. 3(a)) corresponds to the free-slip condition, and $\lambda=0$ (Fig. 3(c)) is the usual noslip condition.

The following numerical results are for a plate withdrawing from a fluid pool (receding contact line), and for contact linedriven spreading of a viscous droplet on a horizontal solid boundary, in the absence of gravity (advancing contact line). Both of these problems have been studied extensively, both experimentally and theoretically (e.g. [7,8,40-45]). To evidence the dependence of the solutions on mesh size, $\Delta$ was varied from $1 / 32$ to $1 / 512$. Note that $\Delta$ represents the maximum mesh resolution on a locally refined adaptive mesh; the interface was always resolved at this maximum resolution. 


\subsection{Withdrawing plate}

Consider a solid plate withdrawing from a square fluid pool of length $L=1$, where $-0.5 \leqslant x, y \leqslant 0.5$. Gravity $g$ acts downwards. The lower fluid is initially 0.4 high and at equilibrium when the left wall begins to withdraw from the pool at a constant velocity $U=1$ (see Fig. 4). Surface tension $\gamma=7.5$ and both fluids have equal density, 1 , and viscosity, 0.25 . The density and viscosity ratios were set to one to minimize the computational cost; a fixed contact angle $\theta=90^{\circ}$ was imposed at the contact line. The corresponding values of $\mathbf{R e}$ and $\mathbf{C a}$ are 4 and 0.03 , respectively. The results were computed to stationary state at a non-dimensional time step of $10^{-5}$, where the non-dimensional time $\tau=t U / L$.

The results when imposing a no-slip boundary condition along the left wall are presented first. As noted before, the numerical model introduces an effective slip which is on the scale of the mesh size. Fig. 5 illustrates the flow fields about the contact line, at different times $\tau$. The streamlines depict the slip of the contact line along the wall, as it evolves to a stationary state by $\tau=1.8$.

Fig. 6 presents the dependence of the time variation of contact line height on mesh spacing; as expected, these curves do not converge as $\Delta \rightarrow 0$. Fig. 7 illustrates the corresponding stationary state interface profiles and further demonstrates the lack of convergence. The logarithmic variation of the stationary contact line location with $\Delta$ is shown in Fig. 8. The height of the contact line increases from 0.55 to 0.82 as $\Delta$ varies from $1 / 32$ to $1 / 512$. To evidence the stress singularity at the contact line, the shear rate $\partial v / \partial x$ along the solid boundary is plotted in Fig. 9; the shear stress at the contact line clearly diverges with mesh refinement.

The flow in the vicinity of the contact line is strongly dominated by viscous effects that are balanced by surface tension, causing the interface slope to vary dramatically near the contact line. To demonstrate the local change of that slope the interface curvature is plotted in Fig. 10 as a function of distance from the wall $x$ (scaled by $\Delta / 2$ ). As illustrated, the curvature increases dramatically as the interface approaches the wall; far from the wall the curvature approaches zero. Fig. 10 also demonstrates the mesh dependence of the interface curvature.

We now turn to results when the Navier-slip model (Eq. (1)) is imposed along the entire solid boundary. We adopted this approach because it has been suggested that using a small slip length results in effective slip only near the contact line [14], which weakens the local singularity and allows the contact line to slip along the solid boundary. The advantage of the Navier-slip model is that it specifies a slip length instead of relying on the mesh-dependent effective slip of the numerical discretization.

We chose a slip length $\lambda=0.001$ that is approximately twice smaller than the finest mesh size, to demonstrate that solutions do not converge when the slip length is not well resolved. Fig. 11 presents the contact line height versus $\Delta$; the shear rates along the solid boundary are plotted in Fig. 12. As expected, the results depend on mesh size, although the dependence appears to weaken as the mesh is refined, suggesting that further reductions in mesh size would lead to convergence. Unfortunately, further refinement would also require excessive computation time.

Finally, recall that contact line height increases logarithmically with mesh refinement when the no-slip condition is applied (see Fig. 6); this is not the case for the Navier-slip condition, as shown in Fig. 11. Also, contact line height decreases when the Navier-slip condition is applied, compared to the no-slip condition. The maximum shear rate at the contact line is also significantly reduced by the slip condition (compare Fig. 12 to Fig. 9). All of this implies that the results obtained when the Navier-slip condition is applied underestimate actual values, unless a very small slip length is employed.

\subsection{Spreading droplet}

The second test is of the spontaneous spread of a viscous droplet. An initially semicircular droplet $\left(\theta=90^{\circ}\right)$ of radius $R=0.5$ is placed on a solid surface in a square domain of length $L=1$, where $-0.5 \leqslant x, y \leqslant 0.5$; for reasons of symmetry only half the droplet is modeled. The droplet moves across the solid surface towards a new equilibrium configuration defined by a change in $\theta\left(=60^{\circ}\right)$. Surface tension $\gamma=7.5$ and both fluids have equal density, 1 , and viscosity, 0.25 . Re and Ca, based on the

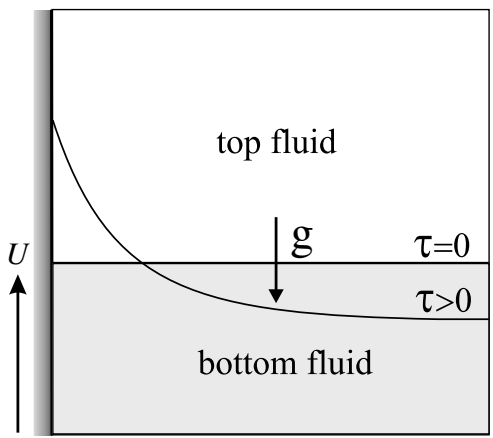

Fig. 4. The withdrawing plate, initially at $\tau=0$, and after the plate begins to move $(\tau>0)$. 

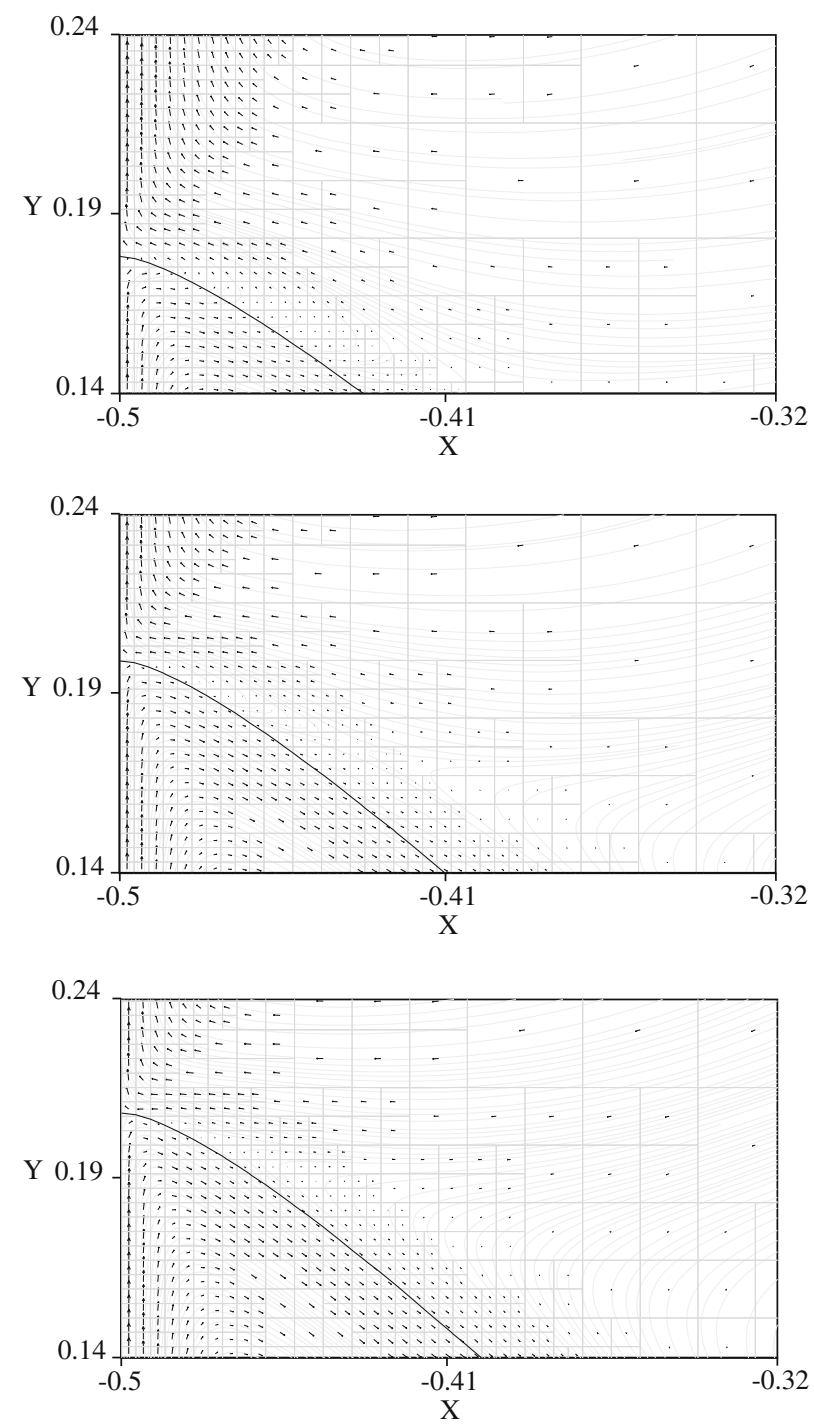

Fig. 5. Velocity fields at $\tau=1,1.4$, and 1.8 (top to bottom), as the contact line moves towards stationary state $\left(\tau=1.8 ; \theta=90^{\circ}\right.$ and $\Delta=1 / 256$ ).

maximum velocity of the flow and the initial radius of the drop $R$, are approximately 2 and 0.03 , respectively. Results are presented at non-dimensional times $\tau=t \mu / \rho R^{2}$; a constant non-dimensional time step of $10^{-5}$ was specified for all cases.

First, the no-slip boundary condition was imposed along the solid boundary, and a fixed $\theta=60^{\circ}$ was specified. The finest mesh size varied from $\Delta=1 / 32$ to $1 / 256$. Fig. 13 provides details of the flow field about the droplet at different times $\tau$ for $\Delta=1 / 256$. The streamlines depict the vortices induced above the droplet. At early times $(\tau=0.01)$, strong flow occurs close to the contact line as the droplet evolves to its equilibrium state. These vortices dissipate at later times as the droplet approaches its equilibrium position.

Fig. 14 shows $r(\tau) / R$, the ratio of the instantaneous droplet radius to the initial droplet radius, at different mesh resolutions, as a function of $\tau$. As shown, $r(\tau) / R$ does not converge with mesh refinement; rather, the contact line velocity is higher on a coarse mesh, which is a consequence of a larger effective slip length; conversely, a finer mesh leads to less slip, which slows the contact line. Fig. 14 also shows that the equilibrium configuration converges with grid refinement. Table 1 presents the convergence of the equilibrium position and shape with grid refinement by comparing the equilibrium volume fraction field with the expected volume fractions (corresponding to a circular segment of the same volume, with $\theta=60^{\circ}$ ):

$$
\begin{aligned}
& L_{1}=\sum_{i=1}^{N}\left|f_{i, \text { exact }}-f_{i, \text { approx. }}\right| \Delta_{i}^{2} \\
& L_{\infty}=\max \left|f_{i, \text { exact }}-f_{i}\right|
\end{aligned}
$$




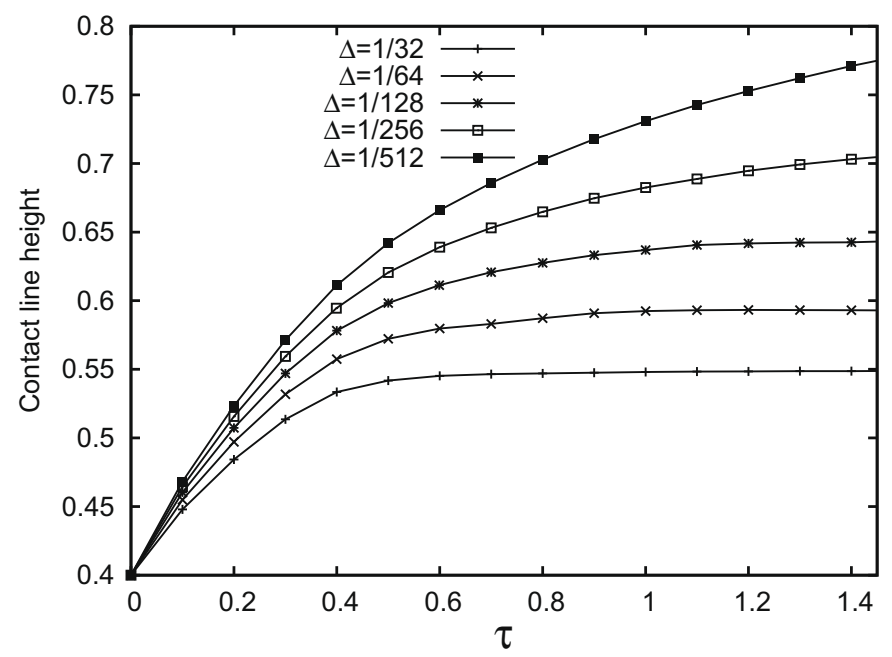

Fig. 6. Dependence of the time-varying contact line position on mesh spacing. A no-slip boundary condition is imposed along the solid surface.

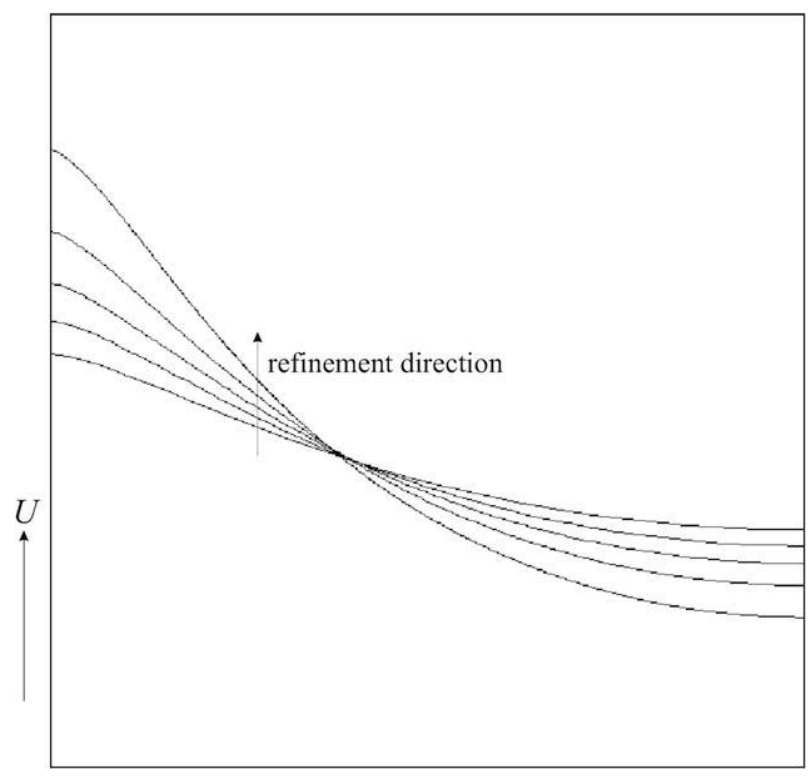

Fig. 7. Stationary state interfaces at different mesh resolutions $\Delta=1 / 32,1 / 64,1 / 128,1 / 256$, and $1 / 512$ (from bottom to top).

$N$ is the number of cells in the domain, and $\Delta_{i}$ represents the size of a cell $i$ in the adaptive mesh. The results show that the mean and maximum errors decrease with mesh refinement. More important, however, is that the maximum errors are well below one, which implies that the equilibrium position and shape are well within a single mesh cell of the exact solution.

To evidence the stress singularity at the contact line, the shear rate along the solid boundary is plotted in Fig. 15 at time $\tau=0.4$. The peak shear rate clearly increases with mesh refinement. Droplet profiles at $\tau=0.4$ are shown in Fig. 16 : the effect of mesh refinement is most apparent near the contact line; the interface profile away from the contact line region is less influenced by the mesh size. These observations are all consistent with the interpretation of an effective slip length.

Figs. 17 and 19 illustrate results when the Navier-slip law, with $\lambda=0.001$, is imposed along the solid boundary. The maximum mesh size varies from $\Delta=1 / 32$ to $1 / 256$. Fig. 17 shows $r(\tau) / R$ at different mesh resolutions. The shear rate along the solid boundary is plotted in Fig. 19 at $\tau=0.4$. Like for the no-slip condition, both $r(\tau) / R$ and the shear rate at the contact line depend on mesh spacing when the Navier-slip condition is imposed.

A grid refinement study is also presented using $\lambda=0.02$, to show that convergence is achieved when the mesh size is smaller than the slip length. Fig. 18 shows that for $\lambda=0.02, r(\tau) / R$ converges with mesh refinement, indicating that one can obtain mesh-independent results when the slip length is resolved. 


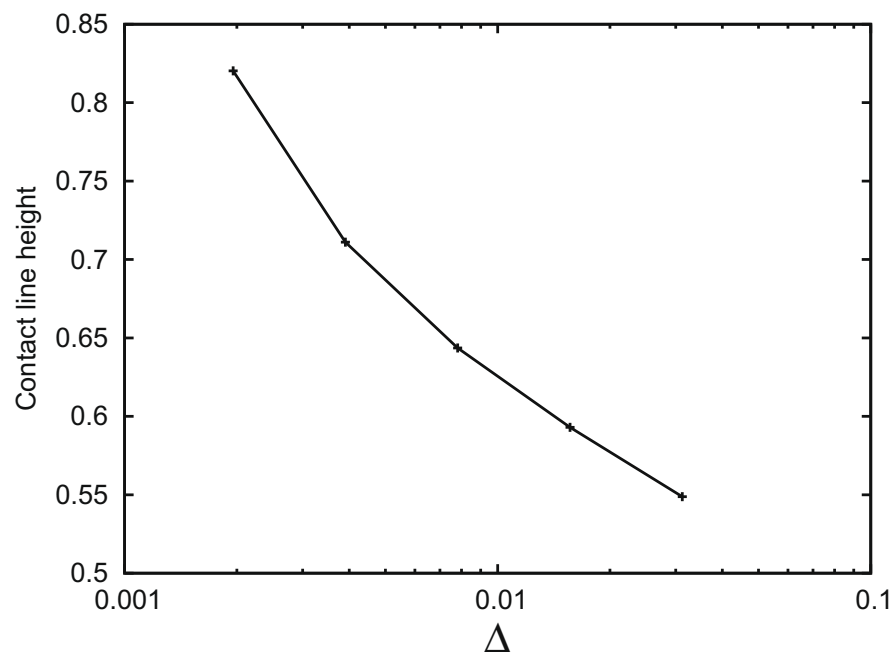

Fig. 8. Dependence of the stationary state contact line height on $\Delta$ : the contact line height increases approximately linearly as the mesh size decreases logarithmically.

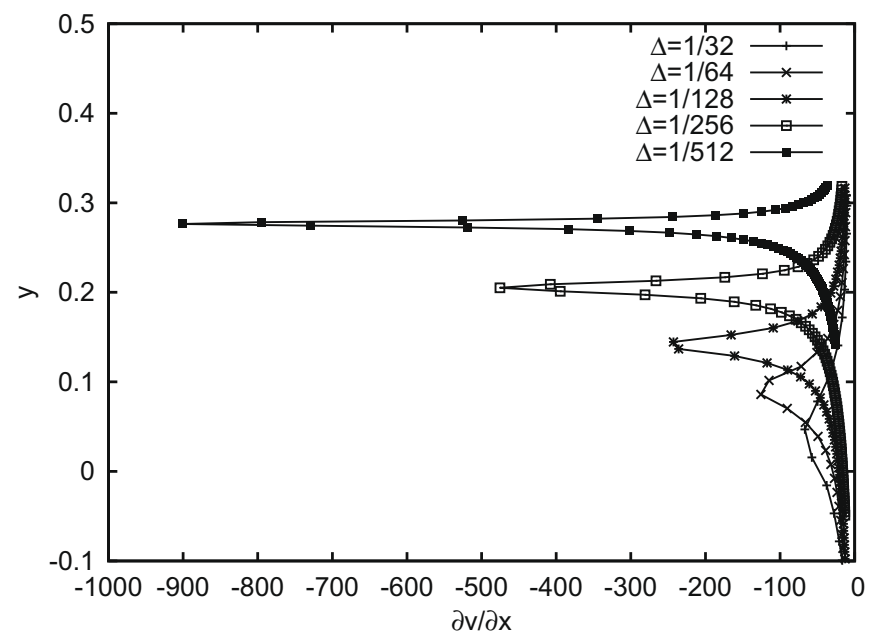

Fig. 9. Stationary state shear rates at different mesh resolutions, for a no-slip boundary condition.

Finally, Voinov [40] presents an analysis of advancing contact lines that predicts a contact line velocity for 2D droplet spreading that behaves as $A / \ln (B / \lambda)$, where $A$ depends on the difference between interface slope away from the contact line and the static contact angle, and $B$ depends on the instantaneous radius of the droplet. The results here are consistent with this theoretical prediction: a larger slip length (on a coarser mesh) leads to an increase of the contact line velocity. This is to be expected, as a larger slip length leads to a decrease in viscous stresses in the vicinity of the contact line (Fig. 15).

\section{A brief review of Cox [13]}

There are two major difficulties that arise when modeling moving contact lines: the specification of $\theta_{d y n}$, and the modification of the no-slip boundary condition to remove the singularity at the contact line. Here we present a contact angle boundary condition derived from fundamental hydrodynamics, that overcomes both of these difficulties in a simple and consistent way. We begin with a review of the analysis of Cox [13], and then present a new numerical contact angle model based on this analysis.

Cox [13] provides a general hydrodynamic description of a moving contact line, that relates $\theta_{d y n}$ to characteristic dimensions of a flow, $u_{c l}$ (or Ca), a contact angle, and $\lambda$ [13]. Beginning from a generalized form of lubrication theory (extended to two fluids), and a general geometry for the system (valid for arbitrary slopes), Cox divides the domain into three distinct regions (illustrated in Fig. 20): (i) an outer macroscopic region of characteristic length $r_{\text {out }}$; (ii) a contact line or intermediate 


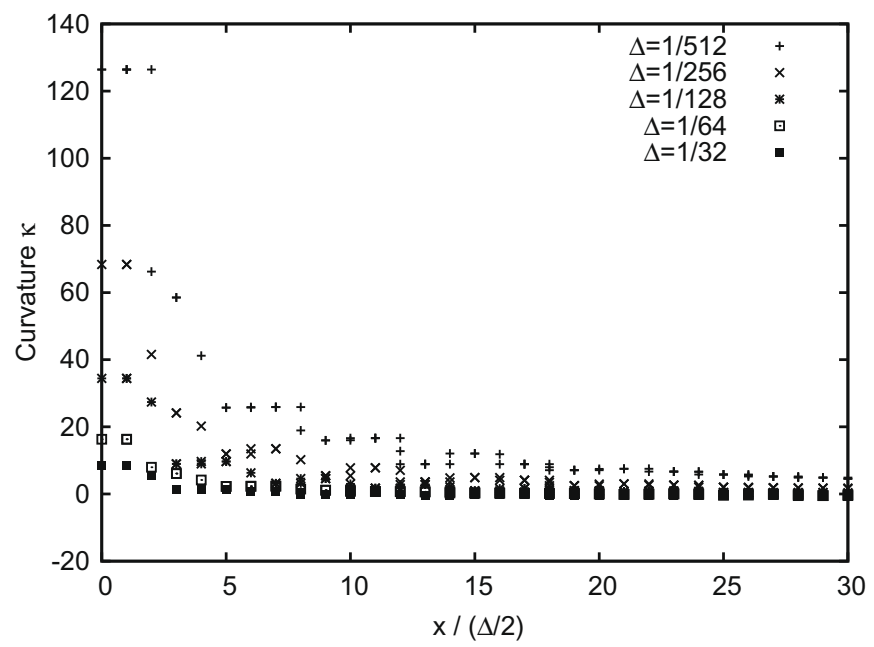

Fig. 10. The variation of the stationary state interface curvature as a function of distance from the wall $x$ (scaled by $\Delta / 2$ ), for a no-slip boundary condition.

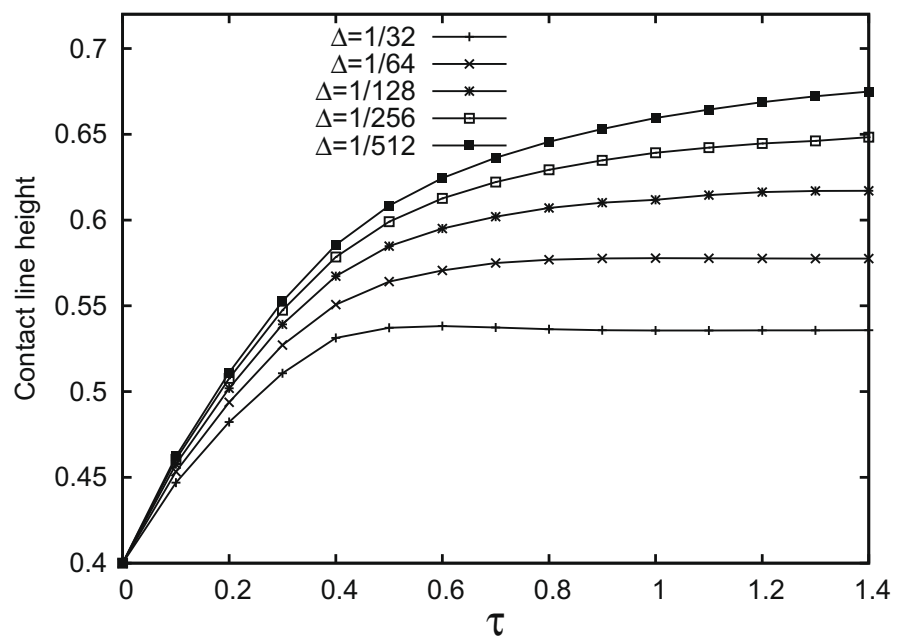

Fig. 11. Dependence of the contact line height on mesh spacing, for the Navier-slip boundary condition, $\lambda=0.001$.

region, over which the interface slope changes sharply, characterized by a balance of viscous and surface tension forces; and (iii) an inner region of characteristic length $r_{i n}$ that is of near molecular size. Let $U$ be the characteristic velocity for the flow when fluid \#2 (of viscosity $\mu_{2}$ and density $\rho_{2}$ ) displaces fluid \#1 (of viscosity $\mu_{1}$ and density $\rho_{1}$ ). During this motion, slip occurs at distances of order $r_{\text {in }}$ so that $\varepsilon=r_{\text {in }} / r_{\text {out }} \ll 1$. Surface tension dominates the flow so that the capillary number $\mathbf{C a}=\frac{\mu_{2} U}{\gamma}$ is small; inertial effects are also negligible, and so the Reynolds number $\mathbf{R e}=\frac{\rho_{2} U L}{\mu_{2}}$ is small.

Cox then uses the solution of the Stokes equations for a wedge flow, and via a double expansion in terms of Ca and $\varepsilon$ matches the solutions in two or three regions of expansion at different distances from the contact line. Cox shows that two regions of expansion are necessary if, as $\mathbf{C a} \rightarrow 0$ and $\varepsilon \rightarrow 0, \mathbf{C a} \ln \left(\varepsilon^{-1}\right) \rightarrow 0$, in which case the interface is almost planar; three regions of expansion are necessary if, as $\mathbf{C a} \rightarrow 0$ and $\varepsilon \rightarrow 1, \mathbf{C a} \ln \left(\varepsilon^{-1}\right)=O(1)$, in which case the angle that the interface makes with the solid surface can change by an amount of order unity.

Fig. 20 illustrates the three regions and the corresponding contact angles: $\theta_{m}$ is the angle in the outer region $r_{\text {out }}$, and $\theta_{w}$ is the angle in the inner region $r_{\text {in }}$ (the angle that a planar interface makes with the solid surface at the contact line). Cox then shows that an asymptotic form of the solution in the outer region $r_{\text {out }}$ written in terms of intermediate variables yields

$$
G(\theta(r))=G\left(\theta_{m}\right)+\mathbf{C a} \ln (r)+\mathbf{C a}\left[\frac{Q_{0}^{*}}{f\left(\theta_{m}, q\right)}\right]
$$

where $q=\mu_{2} / \mu_{1} \cdot Q_{0}^{*}$ must be determined from a particular outer solution and depends on $q, \theta_{m}, d \theta_{m} / d t$, and on the geometry of the outer region, and 


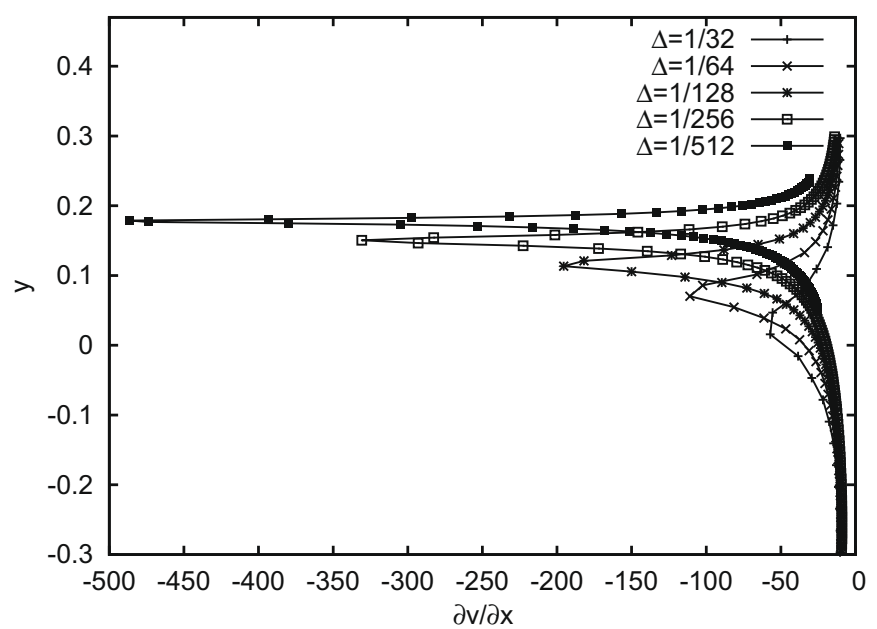

Fig. 12. Stationary state shear rates at different mesh resolutions, for the Navier-slip boundary condition, $\lambda=0.001$.

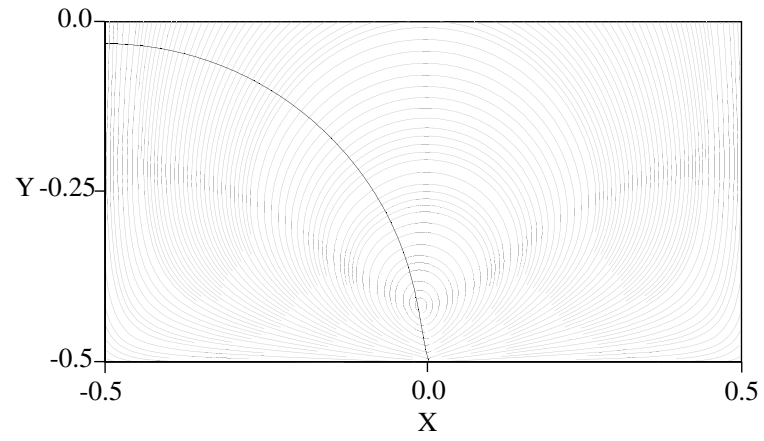

(a)

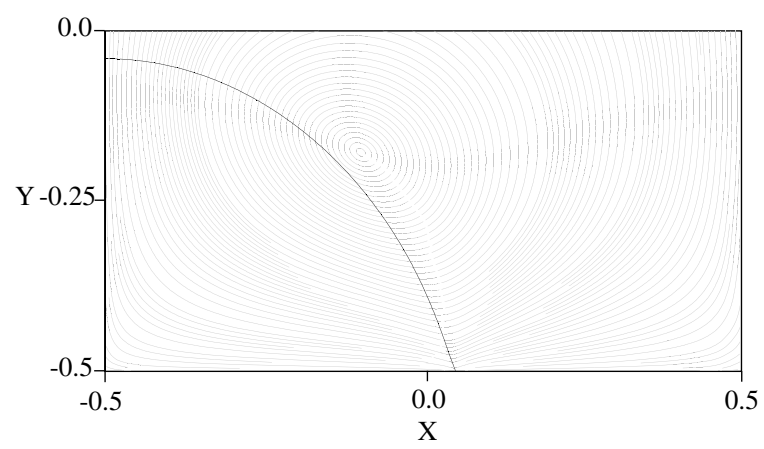

(c)

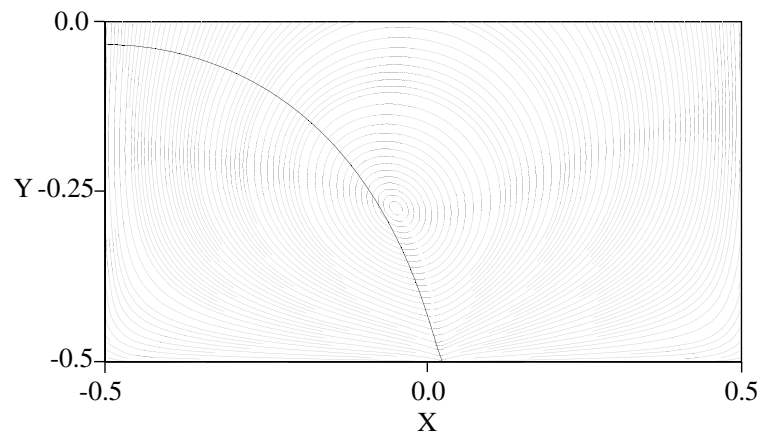

(b)

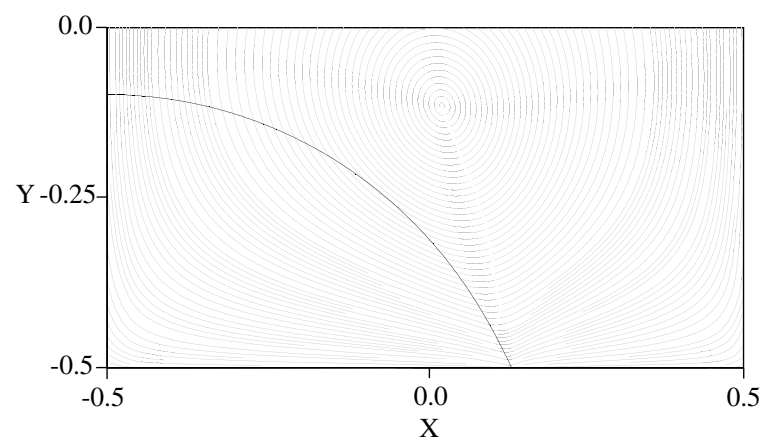

(d)

Fig. 13. Streamlines at $\tau=0.01$ (a), 0.05 (b), 0.1 (c), and 0.4 (d) as droplet moves toward equilibrium state defined by $\theta=60^{\circ} ; \Delta=1 / 256$.

$$
G(\theta)=\int_{0}^{\theta} \frac{d \theta}{f(\theta, q)}
$$

where

$$
f(\theta, q)=\frac{2 \sin \theta\left\{q^{2}\left(\theta^{2}-\sin ^{2} \theta\right)+2 q\left[\theta(\pi-\theta)+\sin ^{2} \theta\right]+\left[(\pi-\theta)^{2}-\sin ^{2} \theta\right]\right\}}{q\left(\theta^{2}-\sin ^{2} \theta\right)[(\pi-\theta)+\cos \theta \sin \theta]+\left[(\pi-\theta)^{2}-\sin ^{2} \theta\right](\theta-\cos \theta \sin \theta)}
$$




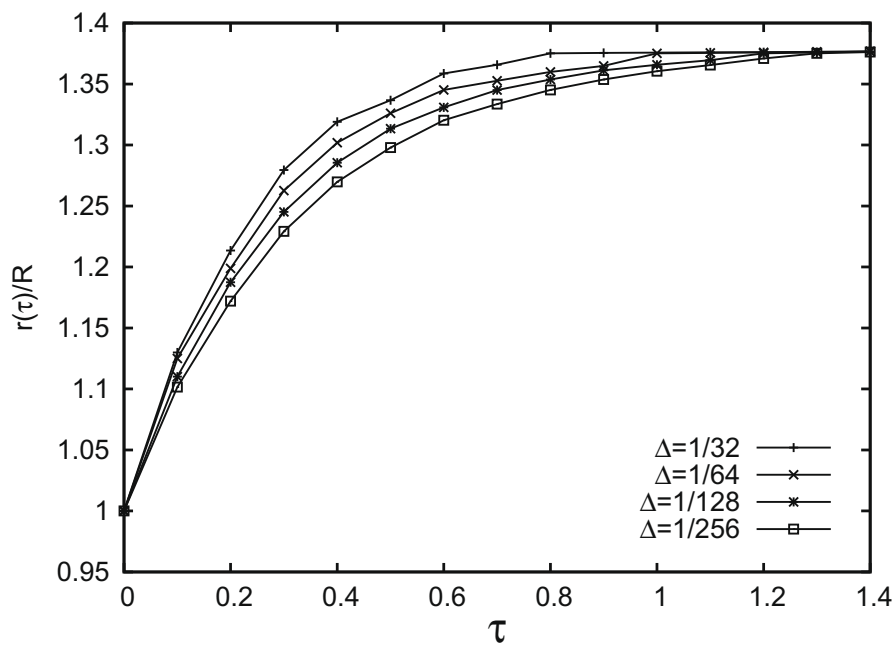

Fig. 14. Dependence of $r(\tau) / R$ on mesh spacing, for a no-slip boundary condition.

Table 1

$L_{1}$ and $L_{\infty}$ norms of errors in volume fractions at equilibrium.

\begin{tabular}{lll}
\hline$\Delta$ & $L_{1}$ & $L_{\infty}$ \\
\hline $1 / 32$ & $3.89 \times 10^{-4}$ & $1.11 \times 10^{-2}$ \\
$1 / 64$ & $1.00 \times 10^{-4}$ & $6.18 \times 10^{-3}$ \\
$1 / 128$ & $3.31 \times 10^{-5}$ & $4.39 \times 10^{-3}$ \\
$1 / 256$ & $6.92 \times 10^{-6}$ & $1.69 \times 10^{-3}$ \\
\hline
\end{tabular}

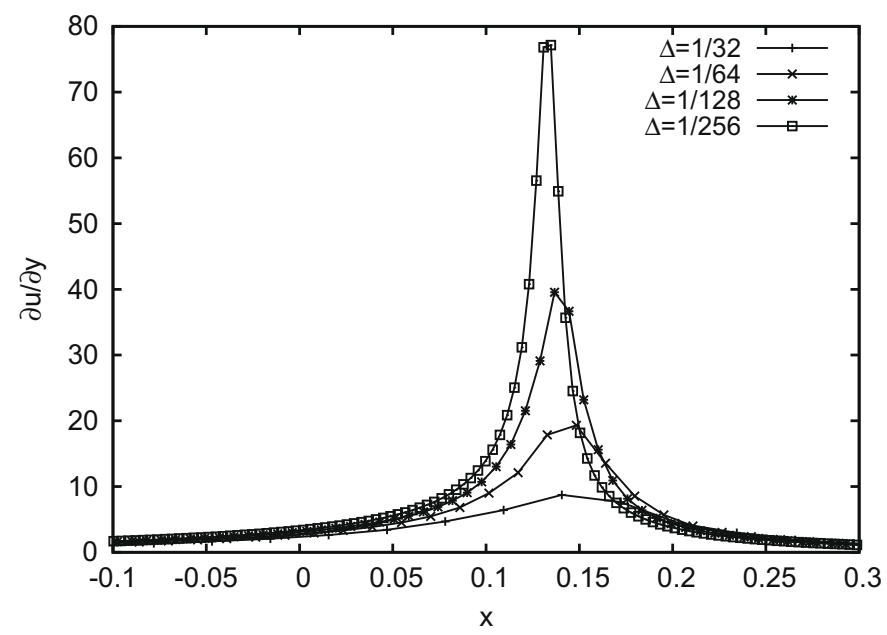

Fig. 15. Shear rate at different mesh resolutions at $\tau=0.4$, for a no-slip boundary condition.

Eq. (9) gives the slope of the fluid/fluid interface at a distance $r\left(r / r_{\text {out }} \ll 1\right)$ using information from the outer region. A similar expansion yields the solution in the inner region $r_{i n}$ in terms of intermediate variables

$$
G(\theta(r))=G\left(\theta_{w}\right)+\mathbf{C a} \ln \left(\varepsilon^{-1} r\right)+\mathbf{C a}\left[\frac{Q_{i}^{*}}{f\left(\theta_{m}, q\right)}\right]
$$

where $Q_{i}^{*}$ is a constant that depends on $q, \theta_{\text {micro }}$, and the specific slip law used. Eq. (12) gives the slope of the fluid/fluid interface at a distance $r\left(r_{\text {in }} / r \ll 1\right)$ using information from the inner region.

Combining Eqs. 9 and 12 yields an expression that relates $\theta_{m}$ and $\theta_{w}$, by matching the two aforementioned solutions in the intermediate region where $\mathbf{C a} \ln \left(r / r_{\text {out }}\right)=O(1)$ : 


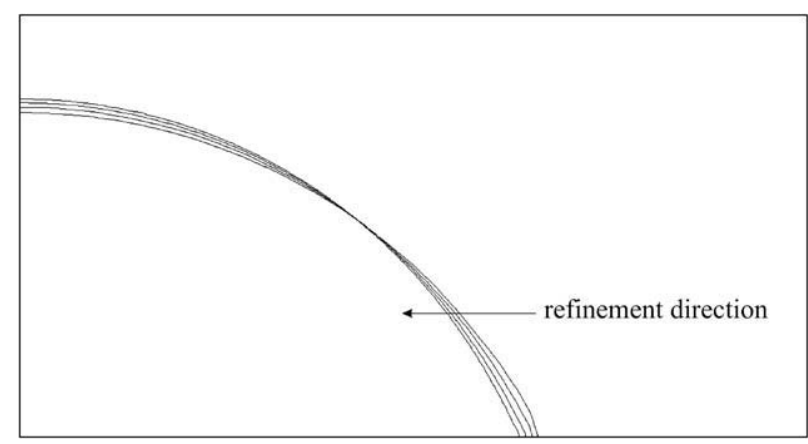

Fig. 16. Droplet shapes at different mesh resolutions at $\tau=0.4$, for a no-slip boundary condition. $\Delta=1 / 32,1 / 64,1 / 128$, and $1 / 256$ (from right to left). The contact line moves from left to right.

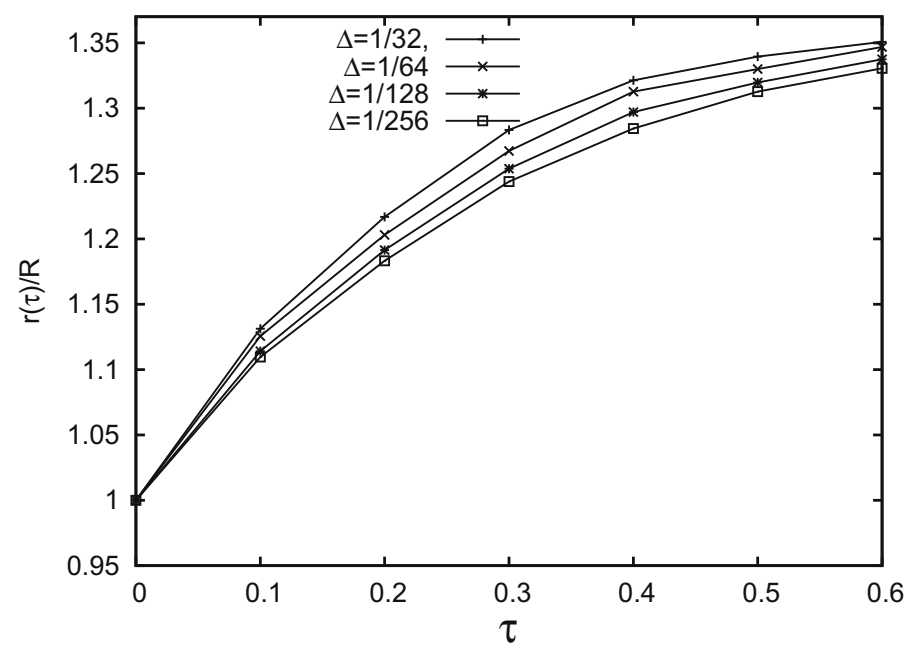

Fig. 17. Dependence of $r(\tau) / R$ on mesh spacing, for the Navier-slip boundary condition, $\lambda=0.001$.

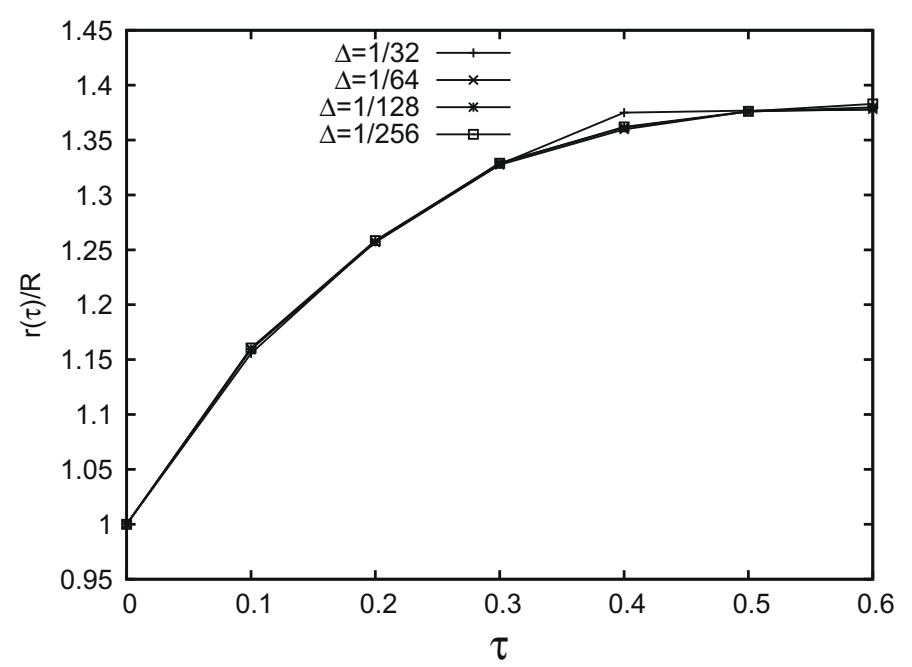

Fig. 18. Convergence of $r(\tau) / R$ with grid refinement is shown for the Navier-slip boundary condition with $\lambda=0.02$. 


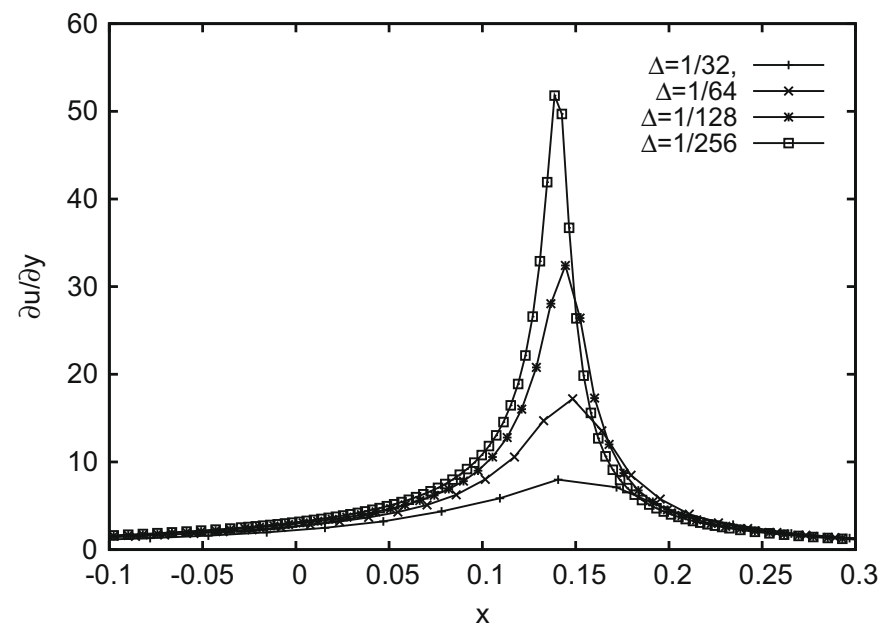

Fig. 19. Dependence of shear rates on mesh resolution at $\tau=0.4$, for the Navier-slip boundary condition, $\lambda=0.001$.

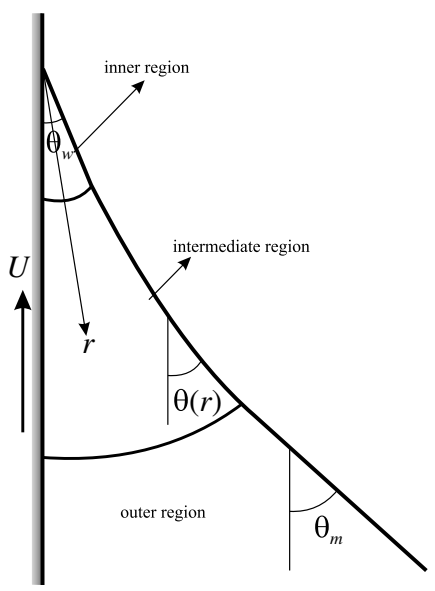

Fig. 20. Outer region: interface slope is $\theta_{m}$; inner region: interface slope is $\theta_{w}$; and intermediate region: interface has a slope $\theta=\theta(r)$ [13].

$$
G\left(\theta_{m}\right)=G\left(\theta_{w}\right)+\mathbf{C a} \ln \left(\varepsilon^{-1}\right)-\mathbf{C a}\left[\frac{Q_{o}^{*}}{f\left(\theta_{m}, q\right)}\right]+\mathbf{C a}\left[\frac{Q_{i}^{*}}{f\left(\theta_{m}, q\right)}\right]
$$

Eq. (13) correct to order $\mathbf{C a}^{0}$ is

$$
G\left(\theta_{m}\right)=G\left(\theta_{w}\right)+\mathbf{C a} \ln \left(\varepsilon^{-1}\right)+O(\mathbf{C a})
$$

Eq. (14) relates the macroscopic and microscopic contact angles, and is only a function of $\theta_{w}$ and the relative length $\varepsilon$, that corresponds to the slip length. However, neither $\theta_{w}$ nor $\varepsilon$ are uniquely defined, in that $\theta_{w}$ can depend on velocity, and $\varepsilon$ is the ratio of $r_{\text {in }}$ to $r_{\text {out }}$ where neither length is a unique quantity. The same equations also result if fluid \#2 recedes, but then $U<0$ and $\mathbf{C a}<0$.

For $\theta_{w} \leqslant \theta_{m}<135^{\circ}$ and $q \ll 0$, Eq. (14) assumes the limiting form

$$
\theta_{m}^{3}=\theta_{w}^{3}+9 \mathbf{C a} \ln \left(\varepsilon^{-1}\right)
$$

This is the so-called "Hoffman-Voinov-Tanner" law [46] that was originally derived from experimental results for small Ca. Also, Sheng and Zhou [29] showed that Eq. (14) can be well approximated by

$$
\cos \left(\theta_{w}\right)-\cos \left(\theta_{m}\right) \simeq 5.63 \mathbf{C a} \ln \left(K / r_{i n}\right)
$$

provided that $q=1$ and $|\cos \theta|<0.6$, where $K$ is a constant (with a dimension of length) that depends on the slip model and the outer region length scale $r_{\text {out }}$. 


\section{A new numerical contact angle model}

The asymptotic matching presented above is the basis of the numerical contact angle model presented here. The theory of Cox relates $\theta$ at various distances; here we draw an analogy to obtain a contact angle boundary condition as a function of mesh size, that yields the same macroscopic contact line configuration.

Fig. 21 illustrates three regions of expansion, corresponding length scales, and contact angles. $r_{o}$ is an outer length scale that could correspond to the length of a domain; $r_{i}$ corresponds to the resolution of the computational mesh; and the length scale at which slip occurs is $\Delta / 2$. By analogy to Cox, $\theta_{a p p}$ corresponds to $\theta_{m}$, and $\theta_{\text {num }}$ (the contact angle boundary condition at the solid wall) corresponds to $\theta_{w}$.

We consider only the leading terms of the asymptotic expansions. Based on Eq. (9), the asymptotic solution in the outer region $r_{o}$ written in terms of intermediate variables yields

$$
G(\theta(r))=G\left(\theta_{\text {app }}\right)+\mathbf{C a} \ln (r)
$$

Similarly, based on Eq. (12), the solution in the inner region $r_{i}$ written in terms of intermediate variables yields

$$
G(\theta(r))=G\left(\theta_{\text {num }}\right)+\mathbf{C a} \ln \left(\frac{r_{o}}{\Delta / 2} r\right)
$$

$\theta_{\text {num }}$ is the angle that the interface makes with the solid surface at a distance on the order of $\Delta / 2$. Combining Eqs. 17 and 18 yields a relationship between $\theta_{\text {num }}$ and $\theta_{\text {app }}$ :

$$
G\left(\theta_{\text {num }}\right)=G\left(\theta_{\text {app }}\right)+\mathbf{C a} \ln \left(\frac{\Delta / 2}{r_{o}}\right)
$$

where the function $G$ is defined by Eq. (10). $\theta_{\text {num }}$ is the mesh- and velocity-dependent geometric boundary condition required for the solution of the macroscopic hydrodynamic equations. $\theta_{a p p}=\theta_{a p p}\left(\mathbf{C a}, \theta_{e}, \ldots\right)$ is the apparent contact angle in the macroscopic region, that we expect to converge to with mesh refinement. This approach to applying a boundary condition at the contact line allows one to use mesh sizes that are very much coarser than the molecular scale, yet obtain realistic results. Finally, note that Cox's theory places no restrictions on the form of the microscopic contact angle, a role played here by $\theta_{\text {num }}$ : it can be constant or velocity-dependent.

$\theta_{a p p}$ in Eq. (19) can be obtained from existing empirical correlations (e.g. [17,46-48]). In most cases described in the literature, $\theta_{a p p}$ is a function of $\mathbf{C a}$ and the equilibrium contact angle $\theta_{e}$. It has been suggested that when viscous drag near the contact line is dominant, then the dynamic behavior of this contact angle versus Ca manifests itself in a "universal" manner [17]: that is, the viscous hydrodynamics near the contact line define the variation of $\theta_{a p p}$ with Ca.

\section{Results}

In the remainder of this paper, we present results using the new boundary condition: $\theta_{\text {num }}$ is prescribed at the contact line, and the no-slip condition is applied along the entire wall. The results demonstrate that the model appears to eliminate the contact line singularity and so yields a well-posed problem that leads to mesh-independent results.

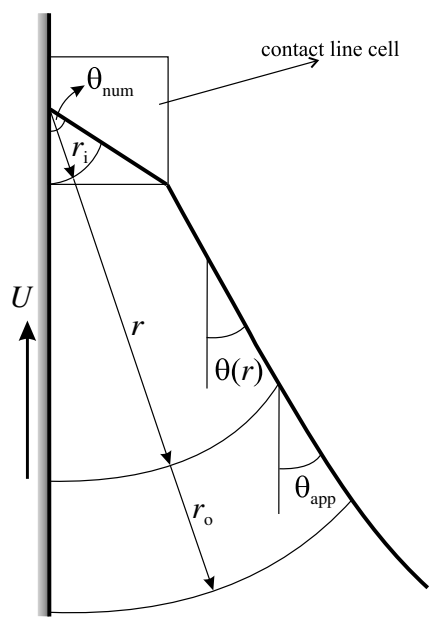

Fig. 21. Outer region: interface slope is $\theta_{a p p}$; inner region: interface slope is $\theta_{\text {num }}$; and intermediate region: interface has a slope $\theta=\theta(r)$. 


\subsection{Withdrawing plate}

To begin, we consider the withdrawing plate of Section 3.1, for which Ca $=0.03$ is a constant, and present results of two sets of simulations. For the first, we calculated a result by applying $\theta_{\text {num }}=90^{\circ}$ on a fine mesh $(\Delta=1 / 512)$; we then used a trial-and-error approach to determine values of $\theta_{\text {num }}$ at other $\Delta$ that yielded very similar stationary state interface profiles. For the second set of simulations, we did the opposite: we calculated a stationary state result by prescribing $\theta_{\text {num }}=90^{\circ}$ on a coarse mesh $(\Delta=1 / 32)$, and then ran many more simulations on finer meshes to determine the values of $\theta_{\text {num }}$ that again yielded very similar interface profiles.

Fig. 22 shows the converged stationary state interface profiles for each of the two sets of results, corresponding to $\theta_{\text {num }}=90^{\circ}, 84^{\circ}, 78^{\circ}, 72^{\circ}$, and $63^{\circ}$ for the first set, and $\theta_{\text {num }}=114^{\circ}, 108^{\circ}, 102^{\circ}, 97^{\circ}$, and $90^{\circ}$ for the second set of simulations. In each case these values correspond to $\Delta=1 / 512,1 / 256,1 / 128,1 / 64,1 / 32$. Of interest is whether the variation of $\theta_{\text {num }}$

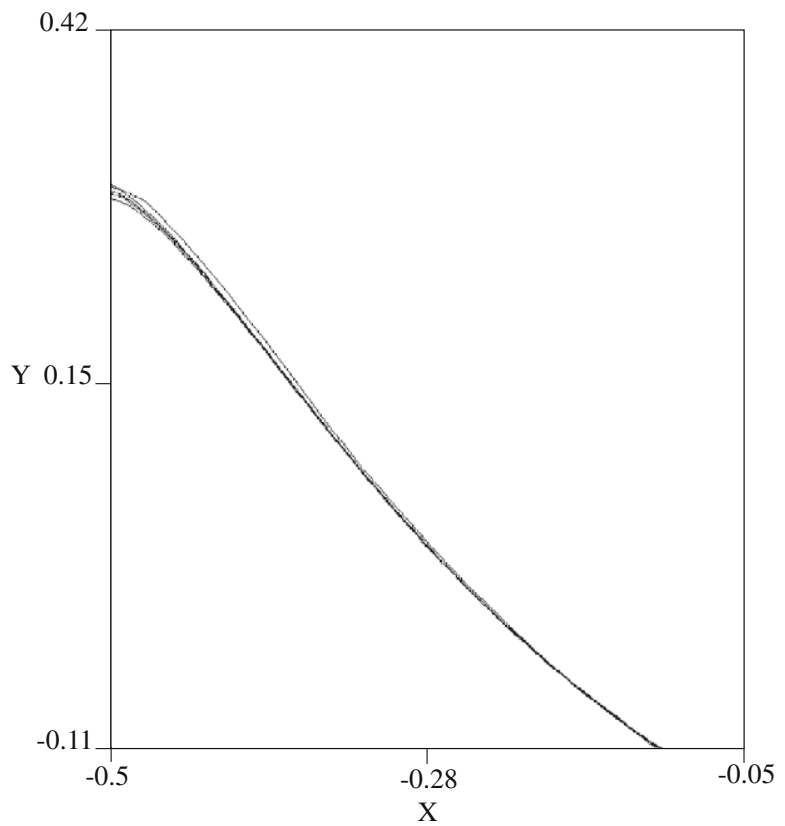

(a)

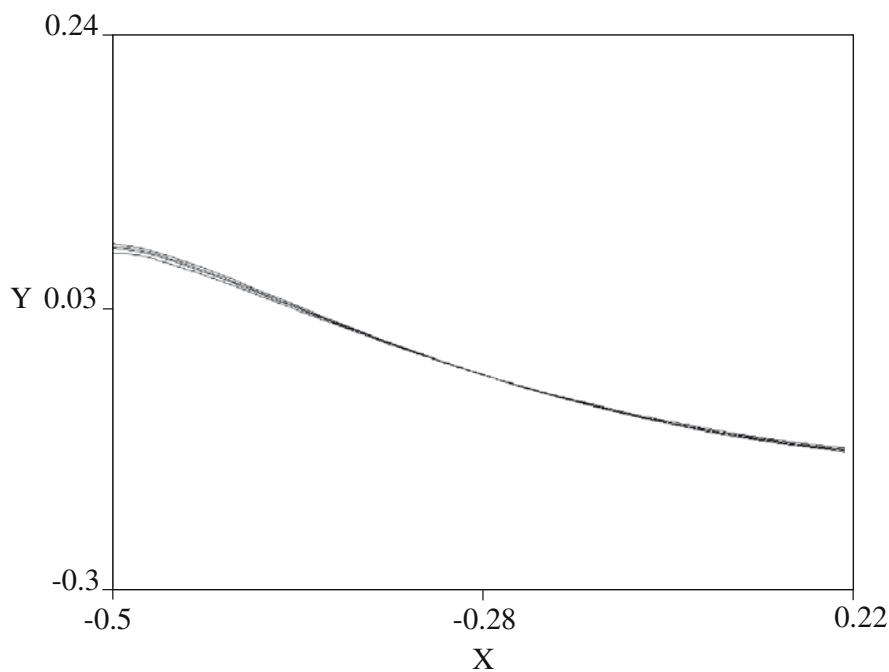

(b)

Fig. 22. Stationary state interfaces magnified near the contact line; $\Delta=1 / 512,1 / 256,1 / 128,1 / 64,1 / 32$. The corresponding values of $\theta_{\text {num }}$ are: (a) $90^{\circ}$, $84^{\circ}$, $78^{\circ}, 72^{\circ}, 63^{\circ}$, and (b) $114^{\circ}, 108^{\circ}, 102^{\circ}, 97^{\circ}, 90^{\circ}$. 
versus $\Delta$ required to obtain this convergence (i.e. to a single $\theta_{a p p}$ ) is of the form of Eq. (19). For simplicity, we used a variation of Eq. (16) to approximate Eq. (19)

$$
\cos \left(\theta_{\text {num }}\right)=\cos \left(\theta_{\text {app }}\right)+5.63 \mathbf{C a} \ln (K /(\Delta / 2))
$$

From the results illustrated in Fig. 22 we measured $\theta_{a p p}$ as the angle between the solid boundary and the interface extrapolated to the solid boundary, as illustrated in Fig. 23. With $a$ as the apex height and $r$ the distance between the planes containing the apex and the contact line, we calculated $\theta_{a p p}=\cos ^{-1}\left[2 r a /\left(r^{2}+a^{2}\right)\right]$.

Fig. 24 illustrates a plot of $\cos \left(\theta_{\text {num }}\right)-\cos \left(\theta_{\text {app }}\right)$ versus 5.63Ca $\ln (K /(\Delta / 2))$. An excellent fit is obtained with $K=0.2$, which is a value also presented in the work of Sheng and Zhou [29]. Fig. 24 clearly shows that the contact angle boundary condition $\theta_{\text {num }}$ scales logarithmically with the numerical slip length. The numerical results, therefore, affirm the validity of the contact angle boundary condition model. Also, as expected, the contact angle increases as the mesh size decreases, thereby allowing the surface tension force to balance the stress singularity. The stationary state shear rate along the solid boundary is plotted in Fig. 25 at different mesh resolutions using the new boundary condition for $\theta_{\text {num }}=90^{\circ}, 84^{\circ}, 78^{\circ}, 72^{\circ}$, and $63^{\circ}$; it is noted that maximum shear rates are about the same as those presented in Fig. 9.

\subsection{Spreading droplet}

Finally we present the results of applying the new mesh-dependent contact angle model to droplet spreading. This time Eq. (20) was used directly to compute the dynamic contact angle boundary condition at the contact line. A fixed $\theta_{a p p}=60^{\circ}$ was set, i.e. $\theta_{\text {num }} \rightarrow 60^{\circ}$ as $\mathbf{C a} \rightarrow 0$, which is the equilibrium contact angle. Unlike the withdrawing plate, $\mathbf{C a}=\mu u_{c l} / \gamma$ is velocity-dependent: $u_{c l}$ is the tangential component of the velocity at the contact line one half cell above the solid surface ( $v_{1}$ in Fig. 3(c)). $K$ was set to 0.02 and $\lambda=\Delta / 2$. The small value of $K$ was chosen to avoid large differences between $\theta_{\text {num }}$ and $\theta_{\text {app }}$, since Eq. (20) is only valid for $|\cos \theta|<0.6$. As noted before, the constant $K$ is not known $a$ priori and depends on the slip

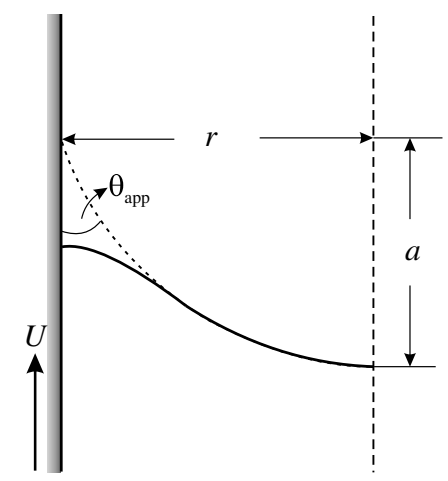

Fig. 23. The apparent contact angle is measured by fitting a circle to the interface and extending it to the solid surface.

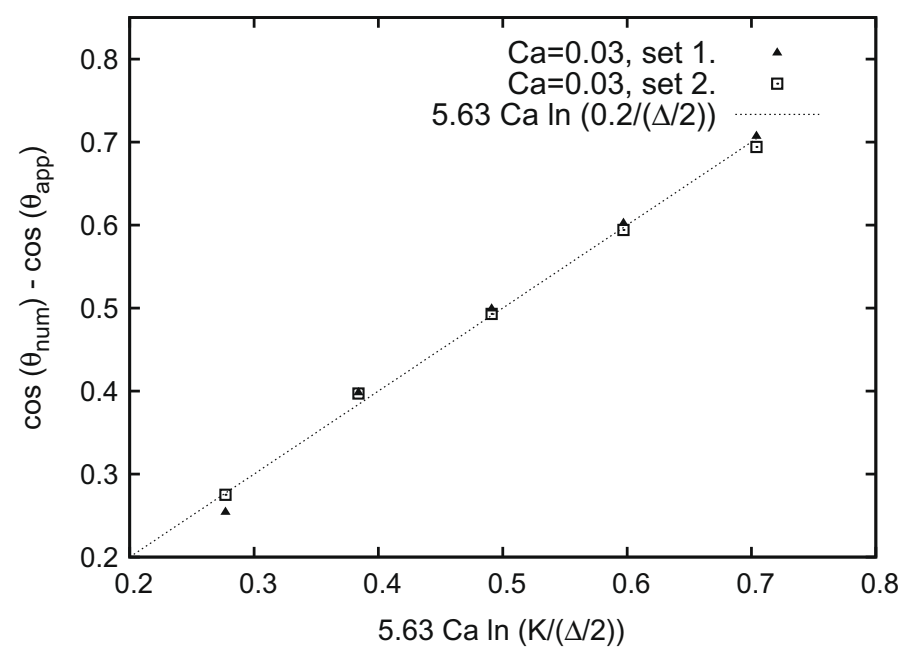

Fig. 24. $\theta_{\text {num }}$ as a function of $\mathbf{C a} \ln (K /(\Delta / 2))$. Filled triangles: $\theta_{\text {num }}=90^{\circ}, 84^{\circ}, 78^{\circ}, 72^{\circ}, 63^{\circ}$; open squares: $\theta_{\text {num }}=114^{\circ}, 108^{\circ}, 102^{\circ}, 97^{\circ}, 90^{\circ}$, where these values correspond to $\Delta=1 / 512,1 / 256,1 / 128,1 / 64$, and $1 / 32$, respectively. The dashed line corresponds to Eq. (16), with $K=0.2$. 
model and the size of the macroscopic region (probably a role played here by the radius of the drop $R$ ). The true value of $K$ could be determined by fitting numerical data to data obtained experimentally or theoretically.

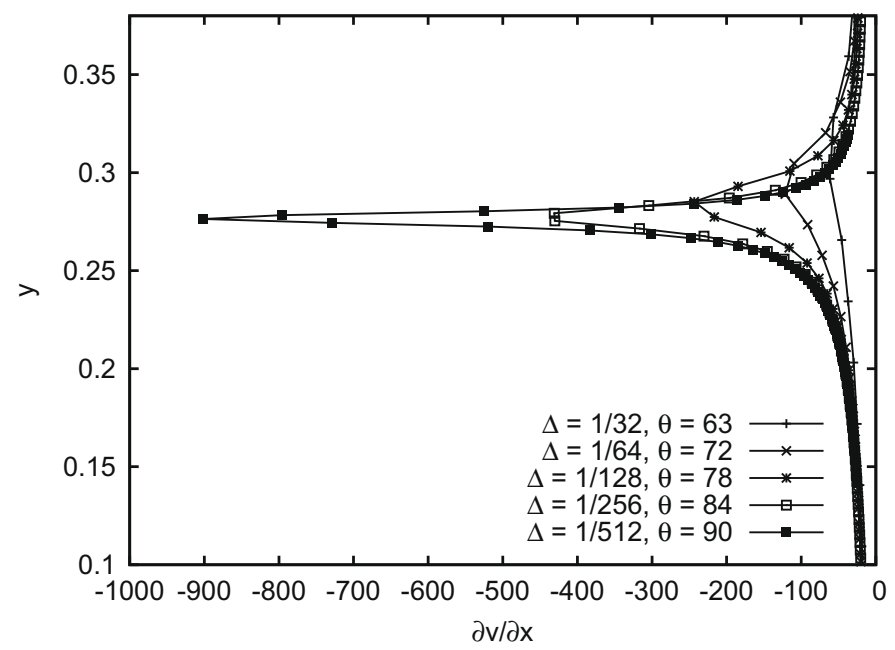

Fig. 25. Stationary state shear rates at different mesh resolutions using the new boundary condition $\theta_{\text {num }}$.

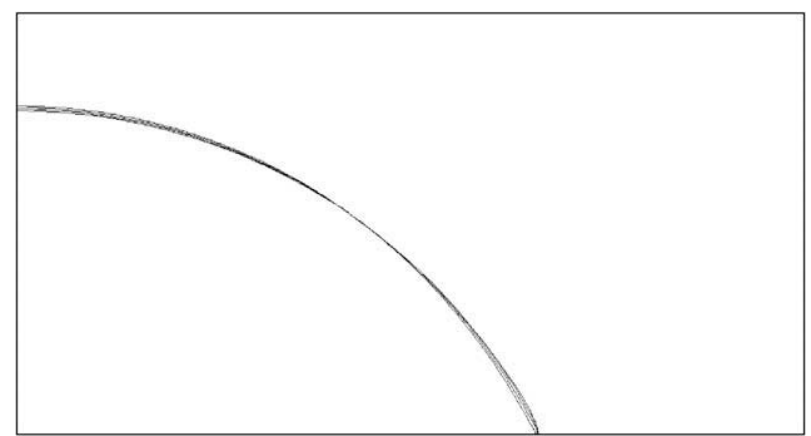

Fig. 26. Droplet shapes converge with mesh refinement. $\Delta=1 / 32,1 / 64,1 / 128$, and $1 / 256$, at $\tau=0.4$. The contact line moves from left to right.

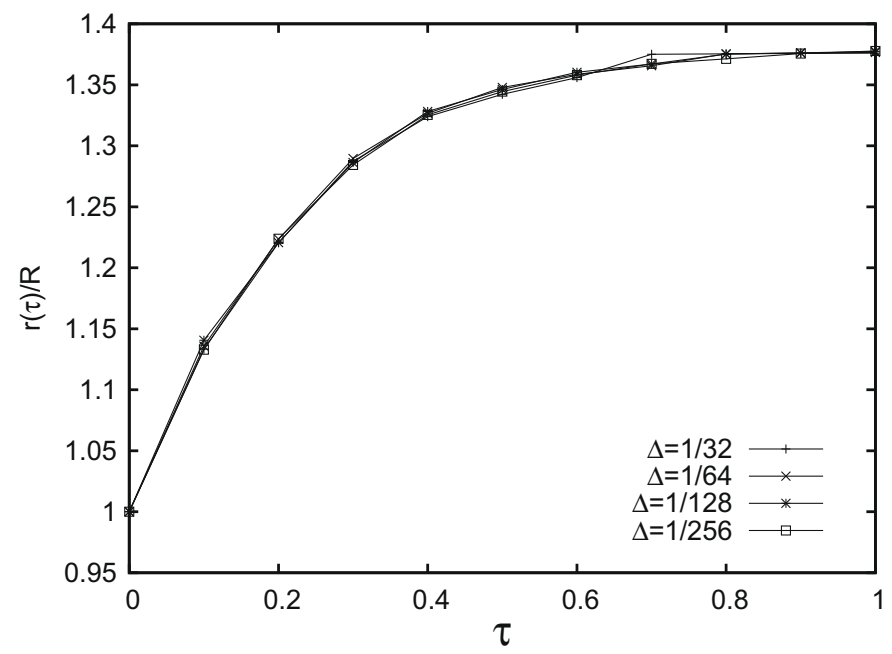

Fig. 27. Convergence of $r(\tau) / R$ with mesh refinement. The dynamic contact angle model is used to compute the contact angle boundary condition $\theta_{\text {num }}=\cos ^{-1}\left[\cos \left(60^{\circ}\right)+5.63 \operatorname{Ca} \ln (0.02 /(\Delta / 2))\right]$. A no-slip boundary condition is imposed along the solid surface. 
Table 2

Droplet radii versus time as a function of mesh refinement. The exact radius corresponding to a circular segment of the same volume with $\theta=60^{\circ}$ is 0.6925 .

\begin{tabular}{lllllll}
\hline$\Delta$ & $\tau=0.2$ & $\tau=0.4$ & $\tau=0.6$ & $\tau=0.8$ & $\tau=1.0$ & $\tau=2.0$ \\
\hline $1 / 32$ & 0.6102 & 0.6619 & 0.6779 & 0.6877 & 0.6879 & 0.6881 \\
$1 / 64$ & 0.6117 & 0.6633 & 0.6799 & 0.6877 & 0.6882 \\
$1 / 128$ & 0.6118 & 0.6640 & 0.6801 & 0.6875 & 0.6887 \\
$1 / 256$ & 0.6119 & 0.6640 & 0.6798 & 0.6860 & 0.6888 \\
\hline
\end{tabular}

Table 3

$L_{1}$ and $L_{\infty}$ norms of errors in volume fractions at equilibrium.

\begin{tabular}{lll}
\hline$\Delta$ & $L_{1}$ & $L_{\infty}$ \\
\hline $1 / 32$ & $4.49 \times 10^{-4}$ & $3.28 \times 10^{-2}$ \\
$1 / 64$ & $1.18 \times 10^{-4}$ & $1.79 \times 10^{-2}$ \\
$1 / 128$ & $3.89 \times 10^{-5}$ & $8.10 \times 10^{-3}$ \\
$1 / 256$ & $1.05 \times 10^{-5}$ & $5.92 \times 10^{-3}$ \\
\hline
\end{tabular}

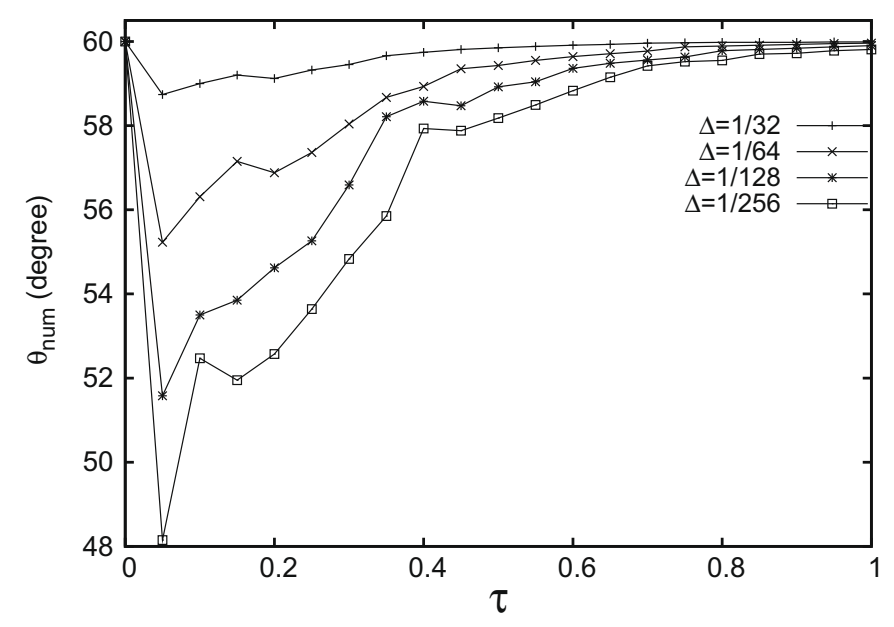

Fig. 28. Variation of $\theta_{\text {num }}$ as a function of $\tau$. Note that $\theta_{\text {num }} \rightarrow 60^{\circ}$ as $\tau \rightarrow \infty$.

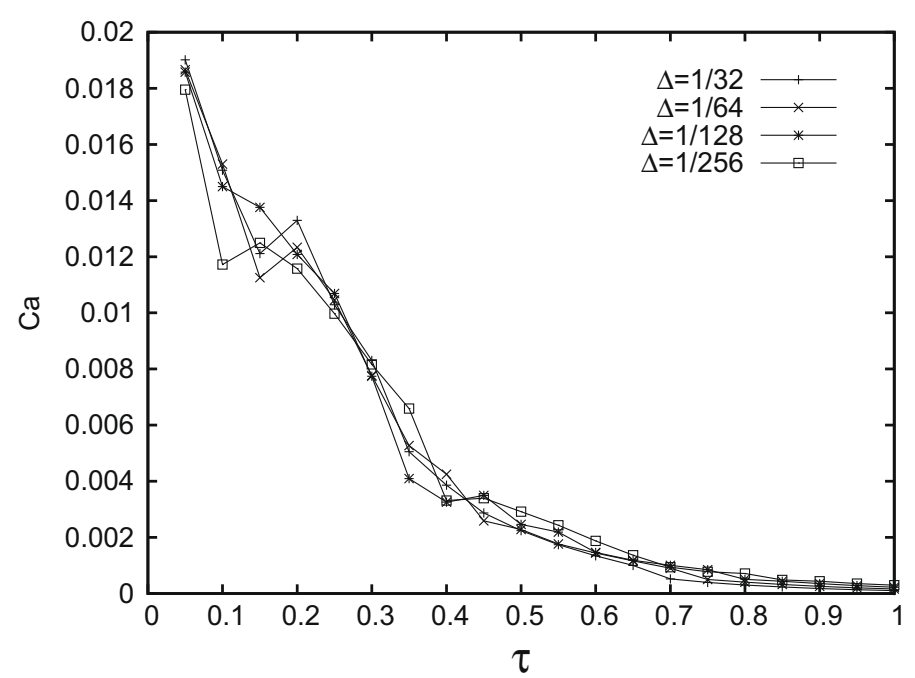

Fig. 29. Variation of $u_{c l}$ (shown in terms of $\mathbf{C a}\left(=\mu u_{c l} / \gamma\right)$ ) as a function of $\tau$. Note that $\mathbf{C a} \rightarrow 0$ as $\tau \rightarrow \infty$. 
Examination of Eq. (20) reveals a gradually varying influence of the contact line velocity on the contact angle model; in particular, estimates of contact line velocities evaluated at finer resolutions are of smaller magnitude than corresponding velocities at coarser resolutions, resulting in smaller contact angles. Eq. (20) also serves as a scaling model for $\theta_{d y n}$ with mesh refinement, which leads to convergence of the numerical solutions. Fig. 26 shows droplet shapes that converge with grid refinement. Fig. 27 demonstrates that with this new dynamic contact angle model, $r(\tau) / R$ converges with mesh refinement. Fig. 27 also shows that the equilibrium configuration converges with grid refinement. Table 2 provides quantitative data of the droplet radii versus time at different resolutions. Table 3 presents the convergence of the equilibrium configuration with grid refinement as measured by the norms defined by Eqs. (7) and (8).

Eq. (20) determines a dynamic contact angle that varies according to mesh size and the contact line velocity. As a result, larger contact angles are computed at coarser resolutions, thus slowing the moving contact lines; the smaller contact angles computed at finer resolutions allow the contact line to speed up. Figs. 28 and 29 demonstrate the variation of $\theta_{\text {num }}$ and the contact line velocity $u_{c l}$ in terms of $\mathbf{C a}\left(=\mu u_{c l} / \gamma\right)$, as a function of non-dimensional time $\tau$, respectively. Fig. 29 illustrates that the contact line velocity is largely independent of mesh resolution, as a result of the dramatic variations of $\theta_{\text {num }}$ illustrated in Fig. 28, especially early in the drop spreading, when the velocities are highest.

\section{Conclusion}

Many numerical solutions of moving contact line phenomena depend on mesh spacing. In particular, one cannot expect the results of simulations to converge when the only slip is the "effective slip" associated with the particular implementation of an algorithm [22,24,25]. Here we presented further evidence of this mesh dependence, when applying both no-slip and Navier-slip boundary conditions. Based on the theoretical analysis of Cox [13], a numerical contact angle model has been developed, that relates $\theta_{\text {num }}$ and $\theta_{\text {app }}$. On the basis of simulations, the linear dependence of $\cos \left(\theta_{\text {num }}\right)-\cos \left(\theta_{\text {app }}\right)$ on Ca $\ln (K /(\Delta / 2))$ is shown. This scaling relationship is then used as a means to evaluate $\theta_{\text {num }}$ as a function of $\theta_{\text {app }}, \mathbf{C a}, \Delta$, and $K$, that yielded mesh-independent solutions of moving contact line phenomena. We showed that our approach is an effective way to deal with under-resolved slip lengths in a consistent manner with hydrodynamics.

\section{Acknowledgments}

This research was supported by NSF-DMS 0405810, CNRS, and NSERC. We thank Stéphane Popinet and Christophe Josserand for helpful discussions.

\section{References}

[1] C. Huh, L.E. Scriven, Hydrodynamic model of steady movement of a solid/liquid/fluid contact line, Journal of Colloid and Interface Science 35 (1) (1971) 85-101.

[2] L.M. Hocking, A moving fluid interface. Part 2. The removal of the force singularity by a slip flow, Journal of Fluid Mechanics 79 (1977) 209-229.

[3] E.B. Dussan V., S.H. Davis, On the motion of a fluid-fluid interface along a solid surface, Journal of Fluid Mechanics 65 (1974) $71-95$.

[4] E.B. Dussan V., E. Rame, S. Garoff, On identifying the appropriate boundary conditions at a moving contact line: an experimental investigation, Journal of Fluid Mechanics 230 (1991) 97-116.

[5] T.D. Blake, M. Bracke, Y.D. Shikhmurzaev, Experimental evidence of nonlocal hydrodynamic influence on the dynamic contact angle, Physics of Fluids 11 (8) (1999) 1995-2007.

[6] J. Billingham, On a model for the motion of a contact line on a smooth solid surface, European Journal of Applied Mathematics 17 (2006) 347-382.

[7] P.G. de Gennes, Wetting: statics and dynamics, Reviews of Modern Physics 57 (3) (1985) 827-863.

[8] L.H. Tanner, The spreading of silicone oil drops on horizontal surfaces, Journal of Physics D: Applied Physics 12 (1979) $1473-1484$.

[9] M.A. Spaid, G.M. Homsy, Stability of Newtonian and viscoelastic dynamic contact lines, Physics of Fluids 8 (2) (1996) $460-478$.

[10] D. Jacqmin, Contact-line dynamics of a diffuse fluid interface, Journal of Fluid Mechanics 402 (2000) 57-88.

[11] V.V. Khatavkar, P.D. Anderson, H.E.H. Meijer, Diffuse-interface modelling of droplet impact, Journal of Fluid Mechanics 572 (2007) $97-127$.

[12] H. Ding, P.D.M. Spelt, Wetting condition in diffuse interface simulations of contact line motion, Physical Review E 75 (2007) 046708.

[13] R.G. Cox, The dynamics of the spreading of liquids on a solid surface. Part 1. Viscous flow, Journal of Fluid Mechanics 168 (1986) 169-194.

[14] P.D.M. Spelt, A level-set approach for simulations of flows with multiple moving contact lines with hysteresis, Journal of Computational Physics 207 (2) (2005) 389-404.

[15] C. Huh, S.G. Mason, The steady movement of a liquid meniscus in a capillary tube, Journal of Fluid Mechanics 81 (1977) 401-419.

[16] E.B. Dussan V., On the spreading of liquids on solid surfaces: static and dynamic contact lines, Annual Review of Fluid Mechanics 11 (1979) 371-400.

[17] R.L. Hoffman, A study of the advancing interface. I. Interface shape in liquid-gas systems, Journal of Colloid and Interface Science 50 (2) (1975) 228235.

[18] J. Fukai, Y. Shiiba, T. Yamamoto, O. Miyatake, D. Poulikakos, C.M. Megaridis, Z. Zhao, Wetting effects on the spreading of a liquid droplet colliding with a flat surface: experiment and modeling, Physics of Fluids 7 (2) (1995) 236-247.

[19] T.A. Baer, R.A. Cairncross, P.R. Schunk, R.R. Rao, P.A. Sackinger, A finite element method for free surface flows of incompressible fluids in three dimensions. Part II. Dynamic wetting lines, International Journal for Numerical Methods in Fluids 33 (3) (2000) $405-427$.

[20] M.A. Walkley, P.H. Gaskell, P.K. Jimack, M.A. Kelmanson, J.L. Summers, Finite element simulation of three-dimensional free-surface flow problems with dynamic contact lines, International Journal for Numerical Methods in Fluids 47 (10-11) (2004) 1353-1359.

[21] M. Bussmann, J. Mostaghimi, S. Chandra, On a three-dimensional volume tracking model of droplet impact, Physics of Fluids 11 (6) (1999) 1406-1417.

[22] M. Renardy, Y. Renardy, J. Li, Numerical simulation of moving contact line problems using a volume-of-fluid method, Journal of Computational Physics 171 (1) (2001) 243-263.

[23] Š. Šikalo, H.D. Wilhelm, I.V. Roisman, S. Jakirli, C. Tropea, Dynamic contact angle of spreading droplets: experiments and simulations, Physics of Fluids 17 (6) (2005) 062103.

[24] J.A. Moriarty, L.W. Schwartz, Effective slip in numerical calculations of moving-contact-line problems, Journal of Engineering Mathematics 26 (1992) 81-86.

[25] O. Weinstein, L.M. Pismen, Scale dependence of contact line computations, Mathematical Modelling of Natural Phenomena 3 (1) (2008) 98-107. 
[26] Y.D. Shikhmurzaev, Singularities at the moving contact line. Mathematical, physical and computational aspects, Physica D: Nonlinear Phenomena 217 (2006) 121-133.

[27] T.D. Blake, in: J.C. Berg (Ed.), Wettability, Chapter 5, Marcel Dekker, New York, 1993.

[28] J. Lowndes, The numerical simulation of the steady movement of a fluid meniscus in a capillary tube, Journal of Fluid Mechanics 101 (1980) 631-646.

[29] P. Sheng, M. Zhou, Immiscible-fluid displacement: contact-line dynamics and the velocity-dependent capillary pressure, Physical Review A 45 (8) (1992) 5694-5708.

[30] M. Latva-Kokko, D.H. Rothman, Scaling of dynamic contact angles in a lattice-Boltzmann model, Physical Review Letters 98 (25) (2007) 254503.

[31] E. Ramé, in: A. Hubbard (Ed.), Encyclopedia of Surface and Colloid Science, Marcel Dekker, New York, 2006, pp. 3602-3618.

[32] I.B. Bazhlekov, A.K. Chesters, Numerical investigation of the dynamic influence of the contact line region on the macroscopic meniscus shape, Journal of Fluid Mechanics 329 (1996) 137-146.

[33] S. Somalinga, A. Bose, Numerical investigation of boundary conditions for moving contact line problems, Physics of Fluids 12 (3) (2000) 499-510.

[34] D. Gueyffier, J. Li, A. Nadim, R. Scardovelli, S. Zaleski, Volume-of-fluid interface tracking with smoothed surface stress methods for three-dimensional flows, Journal of Computational Physics 152 (2) (1999) 423-456.

[35] S. Afkhami. Applying Dynamic Contact Angles To A Three-Dimensional VOF Model. Ph.D. Thesis, University of Toronto, Toronto, 2007.

[36] M. Francois, S.J. Cummins, E.D. Dendy, D.B. Kothe, J.M. Sicilian, M.W. Williams, A balanced-force algorithm for continuous and sharp interfacial surface tension models within a volume tracking framework, Journal of Computational Physics 213 (1) (2006) 141-173.

[37] S. Afkhami, M. Bussmann, Height functions for applying contact angles to 2D VOF simulations, International Journal for Numerical Methods in Fluids 57 (4) (2008) 453-472.

[38] S. Popinet, The Gerris Flow Solver, <http://gfs.sourceforge.net>.

[39] S. Popinet, Gerris: a tree-based adaptive solver for the incompressible Euler equations in complex geometries, Journal of Computational Physics 190 (2) (2003) 572-600.

[40] O.V. Voinov, Hydrodynamics of wetting, Fluid Dynamics 11 (1976) 714-721.

[41] L.M. Hocking, Sliding and spreading of thin two-dimensional drops, Quarterly Journal of Mechanics and Applied Mathematics 34 (1981) 37-55.

[42] E. Ramé, On an approximate model for the shape of a liquid air interface receding in a capillary tube, Journal of Fluid Mechanics 342 (1997) 87-96.

[43] J. Eggers, Existence of receding and advancing contact lines, Physics of Fluids 17 (8) (2005) 082106.

[44] J.H. Snoeijer, Free-surface flows with large slopes: beyond lubrication theory, Physics of Fluids 18 (2) (2006) 02170.

[45] J.H. Snoeijer, G. Delon, M. Fermigier, B. Andreotti, Relaxation of a dewetting contact line. Part 1 . A full scale hydrodynamic model, Journal of Fluid Mechanics 579 (2007) 63-83.

[46] S.F. Kistler, in: J.C. Berg (Ed.), Wettability, Chapter 6, Marcel Dekker, New York, 1993.

[47] T. Jiang, S. Oh, J.C. Slattery, Correlation for dynamic contact angle, Journal of Colloid and Interface Science 69 (1) (1979) $74-77$.

[48] M. Bracke, F. De Voeght, P. Joos, The kinematics of wetting: the dynamic contact angle, Progress in Colloid and Polymer Science 79 (1989) $142-149$. 